\title{
FURTHER OBSERVATIONS AND ANALYSIS OF THE RAPIDLY PULSATING SUBDWARF B STAR EC 20117-4014
}

\author{
S. K. Randall, ${ }^{1}$ G. Fontaine, ${ }^{1}$ S. Charpinet, ${ }^{2}$ A. E. Lynas-Gray, ${ }^{3}$ I. P. Lopes, ${ }^{3}$ S. J. O’Toole,${ }^{4}$ and P. Brassard ${ }^{1}$ \\ Received 2006 January 29; accepted 2006 May 5
}

\begin{abstract}
We present the results of further observations and the subsequent analysis of the rapidly pulsating subdwarf B star EC 20117-4014. Despite our efforts, the only pulsations detected were those already reported by O'Donoghue et al., at $137.3,142.1$, and $157.4 \mathrm{~s}$. We attempted a detailed asteroseismological analysis by thoroughly exploring the fourdimensional parameter space defining our equilibrium stellar structures $\left[T_{\text {eff }}, \log g, M_{*}, \log q(\mathrm{H})\right]$ in search of models that could account precisely for the three observed periodicities. We were able to isolate just two potential families of optimal models, one of which is to be preferred on the basis of the period fit achieved and the surface gravity inferred. To proceed, we used external constraints on the effective temperature from published independent optical spectroscopy. Unfortunately, the contamination of the optical spectrum of EC 20117-4014 made it hard to quantify the uncertainties on the estimated spectroscopic temperature of $\sim 34,800 \mathrm{~K}$, and consequently, we adopted the conservative point of view that the true value would lie somewhere in the rather broad interval 32,800-36,800 K. Despite this limitation, leading to less accurate estimates of structural parameters than has been possible before for isolated pulsating EC 14026 stars, we still find that our asteroseismological exercise has been quite fruitful. Indeed, on the basis of our current models, we find that EC 20117-4014 would have a (logarithmic) surface gravity in the range $5.848-5.864$ dex, a total mass in the range $0.50-0.59 M_{\odot}$, and a (logarithmic) fractional mass of its $\mathrm{H}$ envelope in the interval from -4.10 to -4.25 dex. From these primary parameters, we obtained useful estimates of secondary quantities such as the radius, the luminosity, the absolute visual magnitude, and the distance.
\end{abstract}

Subject headings: stars: individual (EC 20117-4014) — stars: interiors — stars: oscillations — subdwarfs

\section{INTRODUCTION}

Rapidly pulsating subdwarf B (sdB) stars form a relatively new class of variable stars that exhibit low-amplitude, multi-periodic luminosity variations with periods that cover the 80-600 s range, but which are found, more typically, in the 100-200 s interval. They make up only a small fraction $(5 \%-10 \%)$ of the known subdwarf B stars, which are low-mass $\left(\sim 0.5 M_{\odot}\right)$ extended horizontal branch (EHB) objects characterized by a helium-burning core surrounded by a thin hydrogen-rich envelope (Heber 1986). Evolutionary models predict that this envelope is too thin to sustain hydrogen shell burning during helium core burning on the zeroage EHB, which means that instead of ascending the asymptotic giant branch, these objects evolve off the EHB, turn left on the H-R diagram, and join the white dwarf cooling track near $T_{\text {eff }} \sim$ $80,000 \mathrm{~K}$, ultimately becoming low-gravity DAO white dwarfs (Dorman et al. 1993; Bergeron et al. 1994). The core heliumburning phase is identified with subdwarf B stars and lasts around $10^{8} \mathrm{yr}$. Although the basic course of these stars' evolution is well understood, the question that remains is what caused their hydrogen envelopes to become so thin. It is obvious that sdBs must have lost a large fraction of their envelope mass at the time of the helium flash, but the details of this mass loss are unclear. Theories

\footnotetext{
1 Department of Physics, University of Montreal, C.P. 6128, Succursale Centreville, Montréal, PQ H3C 3J7, Canada; randall@astro.umontreal.ca, fontaine@ astro.umontreal.ca, brassard@astro.umontreal.ca.

2 UMR 5572, Université Paul Sabatier et CNRS, Observatoire Midi-Pyrénées, 14 Avenue Edouard Belin, 31400 Toulouse, France; scharpin@ast.obs-mip.fr, lopes@ astro.ox.ac.uk.

3 Department of Astrophysics, University of Oxford, Keble Road, Oxford, OX1 3RH, UK; aelg@astro.ox.ac.uk.

4 Dr. Remeis Sternwarte, Astronomisches Institut der Universität ErlangenNürnberg, Sternwartstrasse 7, 96049 Bamberg, Germany; ai25@sternwarte.unierlangen.de.
}

involving strong stellar winds and various binary evolution scenarios have been brought forward, but still await observational confirmation (e.g., D'Cruz et al. 1996; Sweigart 1997; Han et al. 2002, 2003). It is hoped that this can be achieved on the basis of asteroseismology, the quantitative interpretation of the period spectra uncovered in variable stars.

The idea of pulsating subdwarf B stars was first put forth by Charpinet et al. (1996), who calculated that radial and nonradial pulsations could be excited by an opacity mechanism associated with the presence of iron in the stars' envelopes. At roughly the same time, an independent group of astronomers at the South African Astronomical Observatory (SAAO) discovered the first real sdB pulsator (Kilkenny et al. 1997a), and three others followed shortly (Koen et al. 1997; Stobie et al. 1997; O'Donoghue et al. 1997, hereafter DOD97). Since then, the number of socalled EC 14026 stars (named after the prototype, EC 140262647) has risen to 34, thanks to the efforts of the South African astronomers (e.g., Kilkenny 2002), a Norwegian-German-Italian team (e.g., Silvotti et al. 2002), and a survey carried out by the Montréal group (e.g., Billères et al. 2002), among others. All of the EC 14026 stars discovered to date fall into the well-defined instability strip predicted by theory, most of them clustering around $\log g \sim 5.7$ and $T_{\text {eff }} \sim 33,000 \mathrm{~K}$ (see Fontaine et al. [2006] for a recent review). Subdwarf B stars in this region of the $\mathrm{H}-\mathrm{R}$ diagram attain a balance between gravitational settling and radiative levitation that creates a local overabundance of iron in the driving region of the $\kappa$-mechanism, which results in shortperiod, pressure-mode ( $p$-mode) pulsations. However, not all the stars in the instability strip pulsate. Although the reason for this is not entirely clear, it has been suggested that the presence of stellar winds could have caused a varying degree of mass loss in different stars (Fontaine \& Chayer 1997; see also Chayer et al. 2004). Hence, those objects subject to a stronger stellar wind may 
have lost too much of their iron reservoir to sustain the opacity bump crucial to the driving mechanism. Likewise, it is possible that the constant $\mathrm{sdB}$ stars are simply older and have lost their envelope iron over time (Fontaine et al. 2006).

The rapid pulsators must be distinguished from the more recently discovered long-period variable subdwarf B stars (Green et al. 2003), which oscillate on timescales of the order of 1-2 hr. These significantly longer periods automatically imply high radial order gravity modes, believed to be excited by the same mechanism that drives pressure modes in the fast pulsators (Fontaine et al. 2003). Distinctly cooler than the EC 14026 stars, the slow variables are also more common, accounting for $\gtrsim 75 \%$ of the cool subdwarf B star population (Green et al. 2003).

As the driving mechanism of the instabilities in EC 14026 stars appears to be well understood, stellar modeling can be used to predict the theoretical periods of unstable modes (see Charpinet et al. 2000). As pointed out by Charpinet et al. (2001), the EC 14026 stars tend to exhibit a fair number $(\geq 7-8)$ of pulsations when observed at higher sensitivity than that characteristic of discovery runs. The latter have typically led to the uncovering of 2-4 pulsation modes, generally deemed too few to lead to useful asteroseismological constraints (but see below). Observations with higher sensitivity open up the exciting possibility of employing asteroseismological analyses to derive basic stellar parameters such as effective temperature, surface gravity, stellar mass and, most interestingly, the mass fraction of the hydrogen envelope. Asteroseismological analyses have in fact been completed successfully for four short-period variable subdwarfB stars so far: PG 0014+067 (Brassard et al. 2001; see also Charpinet et al. 2005b), PG 1047+003 (Charpinet et al. 2003), PG 1219+534 (Charpinet et al. 2005c), and Feige 48 (Charpinet et al. 2005a). In each of these cases, the observed periods could be reproduced accurately by just one or two families of models, which were then further constrained on the basis of spectroscopic estimates of the atmospheric parameters. The resulting determination of the total and envelope masses for the four targets is beginning to indicate tendencies that may have a bearing on evolutionary scenarios (see Charpinet et al. 2006); however, it is clear that more EC 14026 stars need to be analyzed for these to become statistically significant.

In this paper, we present further observations and a quantitative analysis of the period spectrum of the fast $\mathrm{sdB}$ pulsator $\mathrm{EC}$ 20117-4014 (DOD97). We review the work already done on this star before presenting an account of our observations and the oscillations extracted from the light curves. We then use these for an exploration into the binary nature of the target. Finally, we describe the results of our asteroseismological analysis and discuss their implications for evolutionary theory.

\section{EC 20117-4014}

EC 20117-4014 was first identified as a bright ultravioletrich star in the Edinburgh-Cape (EC) Blue Object Survey (e.g., Stobie et al. 1992) at a position of $\alpha(1950.0)=20^{\mathrm{h}} 11^{\mathrm{m}} 43^{\mathrm{s}} .8$ and $\delta(1950.0)=-40^{\circ} 14^{\prime} 55^{\prime \prime}$, with an apparent magnitude of $V=$ $12.47 \pm 0.01$ (Kilkenny et al. 1997b). However, spectra taken at SAAO indicate a binary system consisting of a subdwarf B primary and an F or G main-sequence companion (see DOD97 for details), the latter of which contributes more than half of the observed flux in the visual band. The apparent $V$-band magnitude of the sdB component is consequently estimated to be $V=13.55 \pm 0.05$.

In 1997, it was reported that rapid multiperiodic pulsations had been detected in EC 20117-4014, thus making it the fourth member of the then brand new class of EC 14026 stars. In the discovery paper (DOD97) three pulsation periods were identified from nine nights of high-speed photometry gathered over a period of 11 months (34.4 hr of observations altogether): one prominent main oscillation frequency at $f_{1}=7.285 \mathrm{mHz}(137.3 \mathrm{~s})$ with an amplitude of $3.5 \mathrm{mmag}$, as well as two lower amplitude $(\sim 1 \mathrm{mmag})$ pulsations at $f_{2}=7.043 \mathrm{mHz}(142.0 \mathrm{~s})$ and $f_{3}=$ $6.350 \mathrm{mHz}(157.5 \mathrm{~s})$. While the amplitude of the main pulsation frequency remained approximately constant, those of the two secondary periods showed some night-to-night variation. The authors also uncovered a real change in the main oscillation period at $137.3 \mathrm{~s}$ on a timescale of 3 months, an effect they tentatively attributed to the star's motion around the binary system's center of gravity. Finally, there was some weak evidence for a fourth pulsation frequency at $3.58 \mathrm{mHz}(279 \mathrm{~s})$.

DOD97 also obtained numerous spectroscopic measurements of EC 20117-4014 and modeled the spectra as a combination of a subdwarf $\mathrm{B}$ primary and a late $\mathrm{F}$ main-sequence companion. Considerable efforts were invested in this modeling effort aimed at deconvolving the contaminated spectrum and determining as accurately as possible the atmospheric parameters of the $\mathrm{sdB}$ component. Depending on the assumptions made as to the exact nature of the latter, this yielded three sets of estimates for the atmospheric parameters of EC 20117-4014. These are $\log g=$ $5.87 \pm 0.07$ and $T_{\text {eff }}=34,800 \pm 300 \mathrm{~K}, \log g=5.82 \pm 0.17$ and $T_{\text {eff }}=34,400 \pm 700 \mathrm{~K}$, and $\log g=6.19 \pm 0.17$ and $T_{\text {eff }}=$ $36,000 \pm 800 \mathrm{~K}$. The weighted means of these give $\log g=$ $5.90 \pm 0.06$ and $T_{\text {eff }}=34,898 \pm 260 \mathrm{~K}$ as the best currently available estimates. While the formal errors are comparable to what can currently be achieved in the best of cases for isolated $\mathrm{sdB}$ stars (E. M. Green et al. 2006, in preparation), it is clear that they do not take into account systematic effects, possibly quite large, associated with the contamination of the spectrum. Hence, even though the best efforts were invested in the modeling of the polluted spectrum of EC 20117-4014, it is likely that the true uncertainties on the atmospheric parameters of the pulsating component are many times the formal errors and significantly larger than in isolated stars. We discuss below the impact that this extra source of uncertainty has on our asteroseismological analysis.

\section{OBSERVATIONS}

The results presented in this paper are based on high-speed photometry data obtained with the $0.75 \mathrm{~m}$ telescope at SAAO in 2001 and 2002. The observing time allocated consisted of 2 weeks in early 2001 July and another 2 weeks of follow-up time in 2002 mid-July. As our observations were planned entirely around EC 20117-4014, we were able to schedule them according to its best position in the sky, which meant that, theoretically, we could track the star for nearly $12 \mathrm{hr}$ on a clear night. Unfortunately, our original hope of gathering data sets with a markedly improved sensitivity over the original 1997 observations was stifled by poor weather conditions. Indeed, due to highly variable weather during both of the runs, we were able to obtain useful data sets on only five nights in 2001 and on four nights in 2002. Due to passing cirrus clouds and technical problems, some of these data sets show gaps lasting from a couple of minutes to nearly $1 \mathrm{hr}$.

The data obtained at SAAO are supplemented by independent observations carried out on the $1.54 \mathrm{~m}$ Danish telescope at La Silla, Chile, during six nights in 2002 June. Consisting of three shorter runs obtained toward the end of each night, the number of pulsational cycles accumulated is clearly smaller than in the case of the SAAO data; however, the resulting loss of sensitivity is greatly compensated for by the size of the telescope, as well as excellent weather conditions. 
TABLE 1

OBSERVING LOG FOR EC 20117-4014

\begin{tabular}{|c|c|c|c|c|}
\hline Run & $\begin{array}{l}\text { Date } \\
\text { (UT) }\end{array}$ & $\begin{array}{c}\text { Start of Run } \\
\text { (UT) }\end{array}$ & $\begin{array}{l}\text { Sampling Time } \\
\text { (s) }\end{array}$ & $\begin{array}{l}\text { Total Number } \\
\text { of Data Points }\end{array}$ \\
\hline saao-001 .......... & 2001 Jul 6 & $19: 17$ & 10 & 2635 \\
\hline saao-002 ......... & 2001 Jul 10 & $18: 52$ & 10 & 2081 \\
\hline saao-003 .......... & 2001 Jul 11 & $19: 14$ & 10 & 2315 \\
\hline saao-004 .......... & 2001 Jul 13 & $19: 14$ & 10 & 3152 \\
\hline saao-005 .......... & 2001 Jul 14 & $19: 19$ & 10 & 2774 \\
\hline lasilla-001 ....... & 2002 Jun 18 & $07: 23$ & 23 & 499 \\
\hline lasilla- $002 \ldots \ldots$. & 2002 Jun 21 & 07:04 & 23 & 540 \\
\hline lasilla-003 ....... & 2002 Jul 23 & $06: 56$ & 23 & 426 \\
\hline saao-006 .......... & 2002 Jul 17 & $18: 00$ & 10 & 897 \\
\hline saao-007 ......... & 2002 Jul 20 & $19: 30$ & 10 & 3087 \\
\hline saao-008 ......... & 2002 Jul 21 & $17: 07$ & 10 & 2706 \\
\hline saao-009 .......... & 2002 Jul 25 & $17: 11$ & 10 & 3168 \\
\hline
\end{tabular}

A $\log$ of the observing runs can be found in Table 1. In 2001, we gathered $36.0 \mathrm{hr}$ of useful high-speed photometric data over five nights. This corresponds to a duty cycle of $17.9 \%$ and to a temporal resolution of $1.38 \mu \mathrm{Hz}$. The following year at SAAO, we obtained $27.4 \mathrm{hr}$ of data over four nights, corresponding to a duty cycle of $13.6 \%$ and to the same resolution of $1.38 \mu \mathrm{Hz}$. At La Silla, we secured $9.9 \mathrm{hr}$ of photometry, achieving a duty cycle of $8.1 \%$ and a temporal resolution of $2.27 \mu \mathrm{Hz}$.

The SAAO photometry of EC 20117-4014 was obtained with the University of Cape Town (UCT) CCD high-speed photometer, a Wright Instruments Peltier-cooled camera with a $576 \times 420$ thinned, back-illuminated EEV CCD. One of the features of the UCT CCD is that it can be set to run on frame transfer mode, which means that only one-half of the chip is exposed while the other is kept permanently masked off for readout. This implies that there is in effect no readout time, a factor critical to monitoring short-period pulsations. The sampling time at SAAO was hence equal to the exposure time of $10 \mathrm{~s}$ throughout all our runs, while at La Silla the use of a standard $2 \mathrm{~K} \times 4 \mathrm{~K}$ CCD resulted in an effective sampling time of approximately $23 \mathrm{~s}$ compared to an exposure time of $5 \mathrm{~s}$.

In order to account for atmospheric variations, differential photometry was performed at both sites using the only comparison star on the CCD chip, GSC 07952-01358 (Lasker et al. 1990), a star of roughly the same magnitude as EC 20117-4014. Observations taken on the $0.5 \mathrm{~m}$ telescope during the 2001 run at SAAO gave the following magnitude and colors: $V=12.12, B-V=$ $0.88, U-B=0.46, V-R=0.48$, and $V-I=0.98$. Comparing these colors with the calibration of MK spectral types in Allen's Astrophysical Quantities leads us to believe that GSC $07952-01358$ is a main-sequence K-type star. In order to be able to use this red star as a comparison star for the very blue subdwarf, all observations were obtained with a $B$ filter of the Johnson and Bessel variety at SAAO and La Silla, respectively. The two filters were deemed to lie close enough in wavelength so as not to leave their mark on the amplitudes of pulsation.

The SAAO photometry was reduced using DuPhot, a version of the point-spread function-fitting program DoPhot (Schechter et al. 1993), modified for use on the SAAO $0.75 \mathrm{~m}$ telescope by Darragh O'Donoghue. DoPhot is designed to search biassubtracted and flat-fielded digital sky images for any objects above an imposed noise threshold, fit them to a Gaussian, and thus yield their relative magnitudes. The data obtained at La Silla were treated using standard IRAF bias subtraction, flat-fielding, and aperture photometry routines. All flat fields were exposed on the twilight sky. Following the initial reduction, the data were fed into
OSCAR, a special data reduction program developed by one of us (P. B.) and briefly described by Billères et al. (2002). Employing OSCAR allowed us to eliminate low-frequency variations caused by atmospheric effects and differential extinction by subtracting low-order spline functions from the nightly data sets.

However, despite careful data reduction, the variable weather encountered at SAAO left its mark on the quality of the light curves. Detecting rapid luminosity variations of the order of $1 \mathrm{mmag}$ in a $\sim 12.5$ mag star is always going to be a challenge on a $0.75 \mathrm{~m}$ telescope, but observing them through passing cirrus was nearly impossible. Figure 1 shows one of the best segments of the light curve of EC 20117-4014 that we obtained in South Africa. Although the main oscillation of $\sim 137 \mathrm{~s}$ can easily be seen in the figure, it is not as clear as in Figure 2 (bottom) of DOD97, for example. While the light curves from La Silla are of higher quality, this is outweighed by their low duty cycle and temporal resolution. Hence, our data sets are subject to significant levels of noise.

\section{ANALYSIS OF THE LIGHT CURVES}

The time series photometry gathered for EC 20117-4014 was analyzed in a standard fashion using a combination of Fourier analysis, least-squares fits to the light curve, and prewhitening techniques. Since this method has been used for the period determination in numerous EC 14026 stars, we refer the interested reader to a previous paper (Billères et al. 2000) containing a detailed description of the procedure followed.

Fourier transforms (FTs) were calculated for each of the runs listed in Table 1, as well as for the combination of all the nightly light curves obtained in 2001 (SAAO), 2002 June (La Silla), and 2002 July (SAAO). Attempts to combine runs from different seasons and subsequently compute a "grand" FT were unfruitful and hence abandoned. Figure 2 shows one of the best Fourier spectra resulting from a single night's data in the $0-15 \mathrm{mHz}$ bandpass. The part of the spectrum extending beyond $15 \mathrm{mHz}$ and up to the Nyquist limit of $50 \mathrm{mHz}$ is consistent with noise and thus not illustrated here. We can easily identify the three pulsation periods discovered by DOD97. Together with a few spurious low-frequency $(<1 \mathrm{mHz})$ peaks arising from atmospheric variations, they correspond to the only oscillations with amplitudes above the detection threshold (set to 3 times the mean noise level), indicated by the horizontal dotted line. Note that in all SAAO periodograms shown the effects of the $119.7 \mathrm{~s}$ drive error inherent in the $0.75 \mathrm{~m}$ telescope have been removed using prewhitening techniques.

The combined spectra for the 2001 and 2002 runs at SAAO are depicted in Figure 3 (bottom two panels). While the 2002 data in particular are rather noisy, we can nevertheless recover the three basic periods mentioned above in both Fourier transforms. Moreover, the fact that the two sets of data have exactly the same temporal resolution can be exploited to derive a meaningful average spectrum, which is shown in Figure 3 (top). The basic idea behind this procedure is that periodicities present in both data sets will stand out more strongly in the mean Fourier transform, while any spurious peaks, as well as the noise level, should diminish. Indeed, in the resulting periodogram the three previously identified periods stand out more clearly above the noise, leaving no doubt as to their reality.

We show the period spectrum computed from the complete light curve obtained at La Silla in Figure 4. Since the frequency resolution for this run is poorer than for the two sets of SAAO data, it would be imprudent to add the data to the average spectrum calculated earlier. We thus exploit it completely independently, with the aim of later comparing the periodicities extracted to those found at SAAO. Looking at the spectrum, we find that 


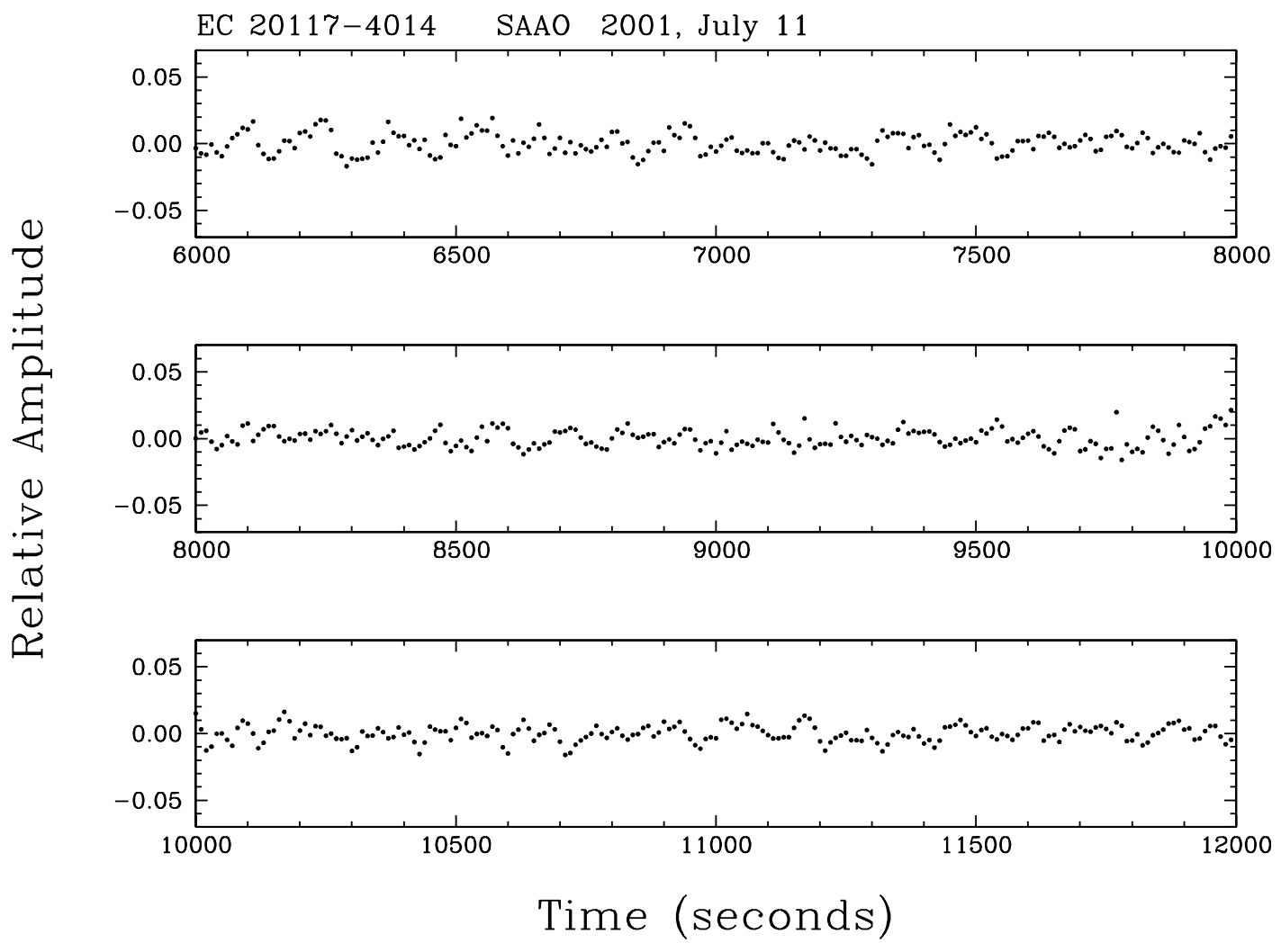

Fig. 1. - Segment of the light curve of EC 20117-4014 from run saao-003, obtained on 2001 November 7, expressed in terms of the residual percentage amplitude relative to the mean brightness of the star. Each plotted point represents a sampling time of $10 \mathrm{~s}$.

just two of the three known periods lie above the formal detection threshold, namely, those at $\sim 137.3$ and $\sim 142.1 \mathrm{~s}$. However, using the fact that the third period, at $\sim 157.4 \mathrm{~s}$, was previously known, we easily recover it in this data set as well, as can be seen in Figure 4. As discussed in Horne \& Baliunas (1986), its statistical significance limit is much lower than for periods for which there is no a priori knowledge, and we consider this recovery real. There seems to be no clear evidence for any further oscillations in the light curve.

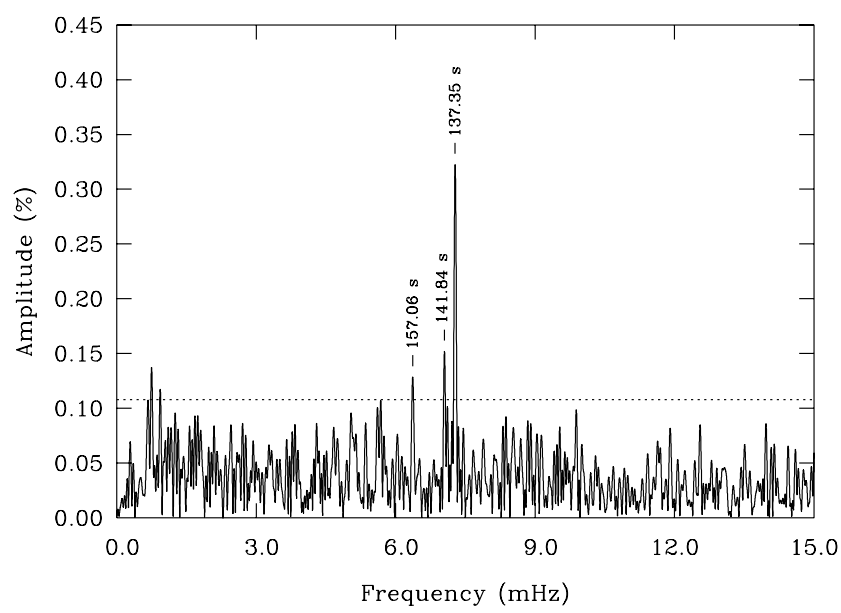

FIG. 2.-The $0-15 \mathrm{mHz}$ bandwidth Fourier amplitude spectrum of the light curve of EC 20117-4014 obtained during run saao-003. The amplitude axis is expressed in terms of the percentage variation about the mean brightness of the star. The positions of the three harmonic oscillations that we identified in the light curve are indicated by short solid line segments. The horizontal dotted line gives the value of 3 times the mean noise level in the bandwidth after removing the three harmonic signals through prewhitening techniques.

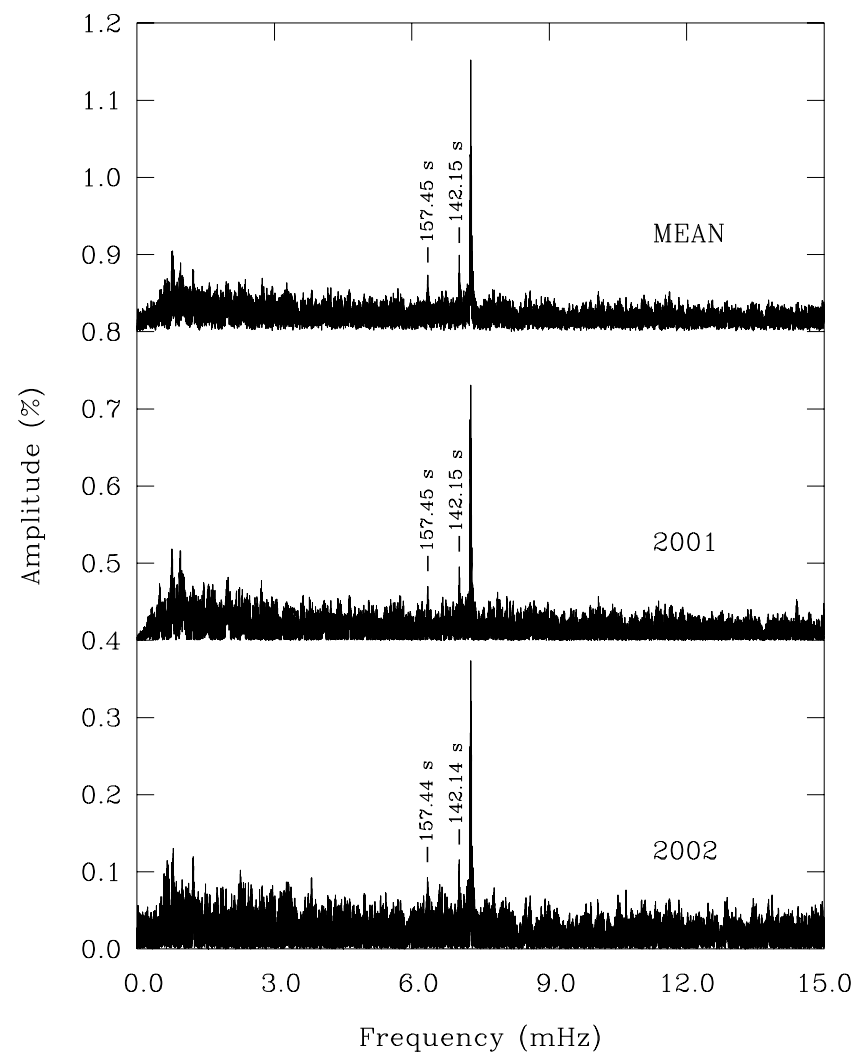

FIG. 3.-Fourier amplitude spectrum $(0-15 \mathrm{mHz}$ bandpass $)$ of the complete light curve of EC 20117-4014 obtained during the 2002 season (bottom) and during the July 2001 season (middle) at SAAO. Some 200,000 frequency points were used in the calculations of these Fourier transforms. Top, Straight mean of those two spectra. Except for arbitrary vertical shifts for the 2002 and mean spectra, the format is the same as that in Fig. 2. 


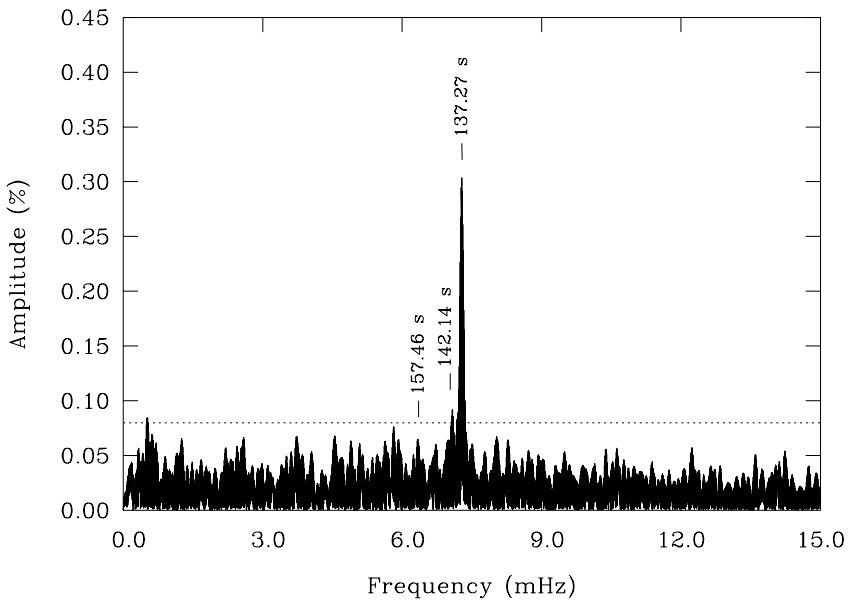

FIG. 4.-Fourier amplitude spectrum $(0-15 \mathrm{mHz}$ bandpass $)$ of the complete light curve of EC 20117-4014 obtained during 2002 June at La Silla. The two harmonic oscillations extracted from the data, as well as the third basic frequency identified from the SAAO data, are indicated by short solid line segments. The format is the same as in Fig. 2.

In order to correctly identify all oscillations present in the light curves, each of the Fourier spectra shown were subjected to prewhitening, a method in which any frequencies present in the transform are identified and then subtracted from the light curve one by one. The results of this exercise are illustrated in Figure 5 for the SAAO 2001 run. The top Fourier transform is that of the original light curve and corresponds to the spectrum shown in Figure 3 (middle), in the region of interest from 6.15 to $7.50 \mathrm{mHz}$. Plotted upside down is the model spectrum, constructed using the three basic periods rising above the threshold in the original transform. The bottom curve illustrates the residual amplitude based on a point-by-point subtraction of the first two spectra in the Fourier domain. Although it is relatively flat and no significant peaks remain, the noise level and low-level fluctuations are too large for us to exclude the possibility of EC 20117-4014 harboring more low-level peaks with amplitudes typical of other well-observed EC 14026 stars. Judging by the fair number $(\gtrsim 7-8)$ of oscillations discovered in other EC 14026 stars when working at higher sensitivity, it seems unlikely that we extracted all the periodicities present. However, the elevated noise level of our spectra prevents us from probing any deeper, leaving only three definite oscillations, the characteristics of which are summarized in Table 2 for all three seasons. We list the frequencies and periods extracted from each of the combined Fourier transforms, together with their amplitudes and phases. Note that while the amplitudes and phases are derived from least-squares fits to the light curve and hence are associated with formal errors, the frequencies are not. This is due to the fact that they are extracted directly from the period spectrum, without any form of fitting procedure taking place. We can, however, estimate the probable accuracy of the frequencies to be of the order of $1 / 10$ of the formal resolution (Billères et al. 2000), and it is these values that are given in the table.

It is interesting to provide a measure of comparison with a different method for estimating the uncertainties on the periods, amplitudes, and phases. To this end, we used the Montgomery \& O’Donoghue (1999) recipes for the 2001 data as an illustrative example. We thus find uncertainties of $0.0006 \mathrm{~s}(0.0022,0.0035 \mathrm{~s})$ on the periods, $0.014 \%(0.014 \%, 0.014 \%)$ on the amplitudes, and $0.9 \mathrm{~s},(3.4,4.8 \mathrm{~s})$ on the phases of the three periodicities with $137.3 \mathrm{~s}$ (142.1, $157.5 \mathrm{~s})$. These are comparable to the entries of Table 2.

Comparing the frequencies extracted from the light curves obtained in different seasons reveals a systematic variation for all

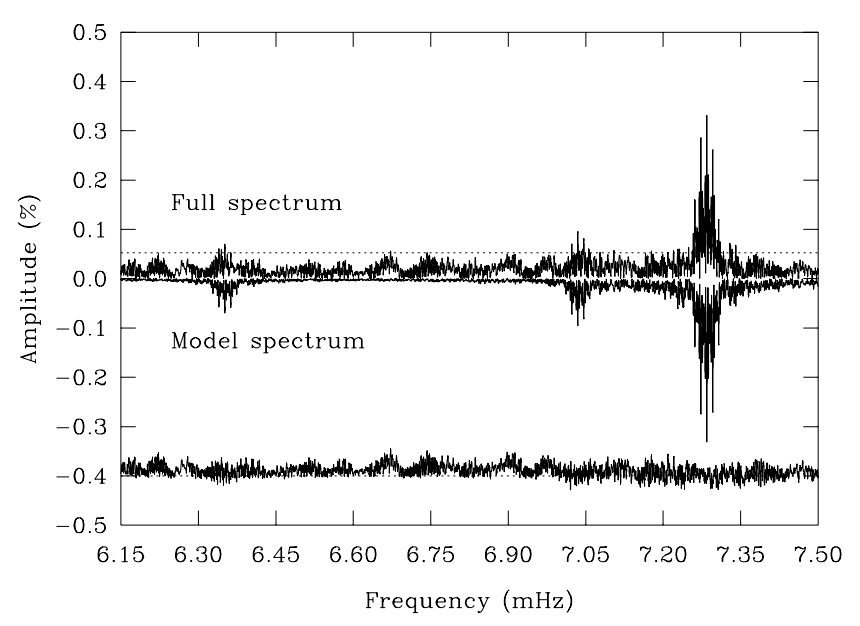

FIG. 5.-Top: Fourier amplitude spectrum of the complete 2001 light curve of EC 20117-4014 in the $6.15-7.50 \mathrm{mHz}$ bandwidth obtained on the basis of 40,000 frequency points. The dotted horizontal line shows the value of 3 times the mean noise level in the bandpass after the prewhitening removal of the three dominant harmonic oscillations. Bottom: Fourier amplitude spectrum (plotted upside down) of the noiseless light curve reconstructed on the basis of the three harmonic oscillations listed in Table 2. We also show the point-by-point frequency difference between the "observed" Fourier spectrum and the "computed" spectrum, but shifted downward by $0.4 \%$.

three harmonic oscillations on a scale larger than the estimated measurement errors. Considering only the higher resolution SAAO data, we find that for each of the periodicities the frequency measured in 2002 is on average $0.6 \pm 0.1 \mu \mathrm{Hz}$ higher than that identified in 2001. This is in line with previous results (DOD97), which reported a frequency change of $1.7 \pm 0.5 \mu \mathrm{Hz}$ in the prominent oscillation over a period of 3 months. The most likely explanation for this frequency shift, as first put forward by DOD97, is orbital motion.

In summary, we detected three harmonic oscillations in the light curve of EC 20117-4014, the frequencies of which seem to vary systematically beyond the estimated measurement errors from one season to the next. It is somewhat disappointing that the oscillations extracted from our three independent sets of data are the same as those already found by DOD97. Moreover, we find no indication of their tentatively identified peak at $\sim 279 \mathrm{~s}$. This may be in line with the fact that modeling predicts that EC 20117-4014, as a rapid pulsator with relatively high values of $\log g \sim 5.9$, should excite modes in the $\sim 60-160$ s period range only (Charpinet et al. 2001). Unfortunately, it also means that the asteroseismological analysis we attempt below is based on only three oscillations, rendering the exercise rather challenging in that, a priori, one could expect a multitude of equally valid solutions. We see below that, due to the relatively high surface gravity of our target star, this is not the case.

\section{EVIDENCE FOR BINARY MOTION FROM FREQUENCY VARIATION}

We believe, following DOD97, that the frequency shifts detected in $\S 4$ are a result of EC 20117-4014's binary motion. This conclusion rests on two observations. First, the frequency variations detected are systematic for all three harmonic oscillations found, implying a global stellar origin, rather than localized stochastic effects. Second, if we take into account the frequencies of the main oscillation measured by DOD97 at $7.28668 \pm$ $0.00014 \mathrm{mHz}$ in $1995 \mathrm{May}$ and at $7.28494 \pm 0.00002 \mathrm{mHz}$ in 1995 August and compare these to the corresponding values in Table 2, we find that the frequency variation is not monotonic 
TABLE 2

Harmonic Oscillations Detected in the Light Curve of EC 20117-4014

\begin{tabular}{|c|c|c|c|c|}
\hline Season & $\begin{array}{l}\text { Frequency } \\
\quad(\mathrm{mHz})\end{array}$ & $\begin{array}{l}\text { Period } \\
\text { (s) }\end{array}$ & $\begin{array}{l}\text { Amplitude } \\
\text { (5) }\end{array}$ & $\begin{array}{l}\text { Phase } \\
\text { (s) }\end{array}$ \\
\hline $2001 .$. & $7.28440 \pm 0.00014$ & $137.2797 \pm 0.0026$ & $0.332 \pm 0.011$ & $45.64 \pm 0.73$ \\
\hline 2002 June .................... & $7.28475 \pm 0.00023$ & $137.2731 \pm 0.0043$ & $0.313 \pm 0.015$ & $100.25 \pm 1.05$ \\
\hline 2002 July..................... & $7.28515 \pm 0.00014$ & $137.2656 \pm 0.0026$ & $0.374 \pm 0.015$ & $40.22 \pm 0.87$ \\
\hline 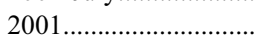 & $7.03472 \pm 0.00014$ & $142.1521 \pm 0.0028$ & $0.094 \pm 0.011$ & $79.38 \pm 2.66$ \\
\hline 2002 June ..................... & $7.03522 \pm 0.00023$ & $142.1419 \pm 0.0046$ & $0.092 \pm 0.015$ & $101.76 \pm 3.76$ \\
\hline 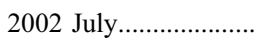 & $7.03526 \pm 0.00014$ & $142.1411 \pm 0.0028$ & $0.117 \pm 0.015$ & $70.72 \pm 2.90$ \\
\hline $2001 \ldots \ldots \ldots \ldots \ldots \ldots \ldots \ldots$ & $6.35136 \pm 0.00014$ & $157.4466 \pm 0.0034$ & $0.073 \pm 0.011$ & $48.63 \pm 3.79$ \\
\hline 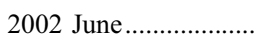 & $6.35100 \pm 0.00023$ & $157.4555 \pm 0.0056$ & $0.064 \pm 0.015$ & $151.77 \pm 5.96$ \\
\hline 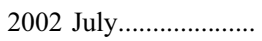 & $6.35186 \pm 0.00014$ & $157.4342 \pm 0.0034$ & $0.088 \pm 0.015$ & $74.00 \pm 4.25$ \\
\hline
\end{tabular}

with time, but instead has exhibited nonlinear changes since the initial measurement.

We attempted to model in a standard manner the frequency variation observed for the dominant oscillation uncovered at $\sim 7.286 \mathrm{mHz}(137.3 \mathrm{~s})$, assuming a circular orbit and representative values for the masses of the components $\left(0.5 M_{\odot}\right.$ for the sdB component and $1.0 M_{\odot}$ for the main-sequence companion). Because of the limited number of sparsely sampled data points (two from DOD97 and three from the present study), we found a large number of acceptable matches to the observed frequency shifts, with a minimum possible period of 18 days and a maximum period of 340 days. Our solutions indicate that there is a strong correlation between the orbital period $P_{\text {orb }}$ and the inclination of the orbital plane $i$, the latter in effect limiting the maximum binary period due to the amplitude of the frequency changes detected. Orbital periods longer than 340 days would require $\sin i>1$ in order to account for the frequency shifts measured and can therefore be excluded. Likewise, a system inclination of much less than $26^{\circ}$ can be ruled out on the basis of a minimum period of 18 days. While the uncertainties on these values are difficult to quantify and depend on the accuracy of the errors on the oscillation frequencies observed, we can conclude that EC 20117-4014 forms part of a binary with a relatively long orbital period in the $\sim 20-350$ day range and a system inclination of $\gtrsim 25^{\circ}$. The former constraint strongly points toward an sdB evolutionary channel involving a stable Roche lobe overflow (already suggested by DOD97); however, more measurements are needed to infer the binary period with any precision.

\section{ASTEROSEISMOLOGY}

\subsection{Initial Considerations}

The ultimate aim of this study is a quantitative asteroseismological analysis of EC 20117-4014 using the "forward approach" pioneered by Brassard et al. (2001) for the rapid pulsator PG 0014+067. In their paper, the authors used 13 observed harmonic oscillations as a basis for finding an optimal model that was capable of reproducing the observed period spectrum surprisingly accurately, as well as predicting another 10 frequencies that were subsequently found in the period spectrum at amplitudes below the detection threshold. Remarkably, the average relative dispersion between the 23 observed periods and those of the corresponding theoretical modes was only $0.8 \%$. Very similar results were obtained for the next two pulsators to be submitted to asteroseismology, PG 1047+003 and PG 1219+534, where 19 and 9 observed periods were fit to predicted modes, with an average dispersion of $0.75 \%$ and $0.6 \%$, respectively (Charpinet et al. 2003, $2005 \mathrm{c})$. In those cases, there were enough observed periods avail- able to constrain fully the models in four-dimensional space (surface gravity, effective temperature, total mass, and $\mathrm{H}$ envelope mass), and complete asteroseismological determinations of these stellar parameters were thus achieved. Typically, only a few welldefined optimal models were found in all of parameter space. What added important credibility to these solutions is the fact that, in each case, the asteroseismological values of $\log g$ and $T_{\text {eff }}$ of one of the optimal models were found to be entirely consistent with completely independent estimates of these parameters from classic time-averaged optical spectroscopy combined with spectral synthesis techniques. A conflict at that level would have cast serious doubt on the validity of the proposed asteroseismological solutions.

A different situation was encountered recently by Charpinet et al. (2005a) in their analysis of the pulsation data of Feige 48, which showed only four independent periods. It was discovered that with only four periods for constraining four-dimensional models, no unique solutions could be found. Instead, two families of solutions emerged (one with a "high" gravity and the other with a "low" gravity), each characterized by the fact that equally valid period fits would be found whatever the value of $T_{\text {eff. }}$ In other words, the effective temperature could not be inferred (within a generous range of possible values) on the basis of asteroseismology alone in that case. As is now well known (see, e.g., Fontaine et al. 1998), the $p$-mode periods in sdB star models are much more sensitive to a change in $\log g$ than to a change in $T_{\text {eff }}$. As a result, asteroseismology leads to much more accurate estimates of $\log g$ than optical spectroscopy can for these stars, while the converse is true for $T_{\text {eff }}$ (see Charpinet et al. [2005a], [2005c] for further discussion of this). Charpinet et al. (2005a) exploited this fact in their analysis of Feige 48: they first used the estimate of $\log g$ from their independent spectroscopy to discard the low-gravity family of asteroseismological solutions and then they used their spectroscopic temperature to pin down the other stellar parameters along the selected high-gravity family of asteroseismological solutions. By further using the spectroscopic uncertainties on $T_{\text {eff }}$ they were able to estimate uncertainties on the other parameters (surface gravity, total mass, and mass of the $\mathrm{H}$ envelope) given by the asteroseismological analysis. The drawback of this approach is that spectroscopy - which is now an intrinsic part of the method - can no longer be used as an independent check on the asteroseismological analysis. But this is the price to pay when there are only a few available observed periods. Furthermore, Charpinet et al. (2005a) easily recognized that their finding of an average dispersion between the observed periods and the theoretical ones at an unprecedented accuracy of $\sim 0.005 \%$ was due mostly to the fact that only four periods had to be fitted, as opposed to nine or more in the previous cases. There is less 
difficulty (and therefore less merit!) in fitting four periods simultaneously than, say, 10. Therefore, Charpinet et al. (2005a) concluded that there is ample room for improvement in the sdB star models currently in use, until simultaneous fits to all periods at a high level of accuracy are reached for all stars investigated.

Our attempt at interpreting the period spectrum of EC 201174014 quantitatively is similar to the analysis of Feige 48 in that we do not have a large number of observational frequencies available. In fact, the outlook is slightly bleaker than for the previous case, as in theory fitting only three experimental periodicities should induce strong degeneracies when attempting to find four free parameters. In practice, however, the stellar parameters specified are not completely independent of each other, and a meaningful fit may nonetheless be achieved. Fortunately for our purposes, EC 20117-4014 appears to be one of the rapid pulsators with the highest surface gravities, at $\log g \sim 5.9$. In such cases, the density of theoretical modes in a given period window is relatively low. Moreover, according to radiative levitation theory, the high gravity causes the partial draining of the iron reservoir in the driving region, leaving a weakened opacity mechanism able to drive fewer modes with smaller growth rates than in less compact EC 14026 pulsators (Fontaine et al. 1998). The number of modes excited (and the density of the period spectrum in general) is therefore relatively low, enabling the determination of a unique solution on the basis of fewer observational periods than was the case for previous asteroseismic targets. As we show below, however, it is the large unquantified uncertainties associated with the spectroscopically derived atmospheric parameters that will ultimately limit our application. Indeed, as was found in the case of Feige 48, there are not enough periods here to uniquely constrain an asteroseismological solution, and the inferred parameters will have to depend on the reliability of the assumed effective temperature given by spectroscopy. With hindsight, it is now clear that with a contaminated optical spectrum and very few modes, EC 200174014 was not the best target that could have been chosen. But, of course, when the target was selected and observed, we did not have the experience and knowledge that we now have.

\subsection{Methodology}

Our approach to asteroseismology consists of searching for theoretical models whose period spectra can closely reproduce the pulsation properties observed in EC 20117-4014. These are found in a double-optimization procedure that simultaneously considers the period matching and model parameter space domains. The first optimization determines the mode identification that yields the best global match between the periods observed and those calculated for a particular model and quantifies the fit in terms of the goodness-of-fit merit function

$$
S^{2}=\sum_{i=1}^{n}\left(P_{\mathrm{obs}}^{i}-P_{\text {theo }}^{i}\right)^{2},
$$

where $P_{\text {obs }}^{i}$ is one of the $n$ periodicities observed and $P_{\text {theo }}^{i}$ is the theoretical period that matches it best. By dividing $S^{2}$ by $n$ and then taking the square root, one obtains the standard deviation between the observed and the assigned periods. Hence, the chosen merit function measures the goodness of fit in the sense of a standard deviation. Models that result in a smaller value of $S^{2}$ provide a better match to the observed pulsations than those with a larger merit function. Note that at this point the period match quantified by $S^{2}$ does not necessarily have to be good; the value of $S^{2}$ simply indicates the best fit that can be achieved to the observed periods on the basis of a given model. It is the goal of the second optimization to determine which model (or models) in parameter space minimizes the merit function and as such optimally reproduces the observed periods.

We currently possess two independent sets of numerical tools that are able to carry out the double-optimization procedure described. The first of these was developed in Montréal and corresponds to an updated version of the code employed by Brassard et al. (2001) in their pioneering asteroseismological analysis of PG 0014+067. The second double-optimization package is operational in Toulouse and was used for the analysis of PG 1219+ 543 and Feige 48 (see Charpinet et al. 2005a, 2005c). Although the two double-optimization packages are numerically independent, they are based on the same theoretical period spectra and yield compatible fits to the observed periods, as they should (see, e.g., Charpinet et al. 2005b for a reanalysis of PG 0014+067 with the Toulouse algorithm). The computation of the period spectra to be matched to the observations involves three codes, starting with the equilibrium model-building algorithm first written by Brassard \& Fontaine (1994) for white dwarfs and later adapted for sdB stars (see, e.g., Charpinet et al. 1997, 2001). These socalled second-generation stellar models require four input parameters, the effective temperature, $T_{\text {eff }}$, the logarithm of the surface gravity, $\log g$, the total mass of the model, $M_{*}$, and the logarithmic depth of the transition between the hydrogen-rich envelope and the helium core $\log q(\mathrm{H})=\log \left[M(\mathrm{H}) / M_{*}\right]$. They constitute spherical static structures, where the central nucleus (comprising around $10 \%$ of the star's mass) has been replaced with a "hard ball." Since the $p$-mode periods that we are interested in correspond to shallow-envelope modes and are completely insensitive to the structure of the nucleus, this is a reasonable approximation to make. Another noteworthy characteristic of the models is that they feature a nonuniform iron abundance profile as a function of depth. The latter is the result of the competing action between radiative levitation and gravitational settling and is characterized by a local enhancement of iron in the driving region. It is this iron enhancement that provides the necessary extra opacity for the $\kappa$-mechanism to operate, thus leading to the excitation of the oscillations observed. Hot subdwarf models must therefore include radiative levitation if any predictions are to be made as to the stability of modes. Moreover, it was shown by Charpinet et al. (2005a; see also Fontaine et al. 2006) that the depth-variable chemical composition of a model affects its eigenfrequencies at the quantitative level, rendering an accurate description of utmost importance when attempting asteroseismology.

In the second step of the period computation process, the equilibrium models are subjected to adiabatic pulsation calculations with the aid of an updated version of the finite-element code described by Brassard et al. (1992). Numerically solving the four adiabatic oscillation equations given, for example, by Unno et al. (1989), the program provides necessary estimates of the eigenmodes that are subsequently used as first guesses for the solutions of the six complex equations describing nonadiabatic nonradial oscillations. The latter are fully solved by an improved version of the nonadiabatic code briefly described by Fontaine et al. (1994). In addition to improved frequencies, the nonadiabatic code provides information on the stability of an eigenmode, which is important for comparing with observations. A typical output from the program is illustrated in Table 3 . While it specifically refers to a given model (see below), we would like to simply illustrate the pulsational properties generated by the code at this point. For each mode identified in a selected period range, Table 3 lists the degree index $l$ (the values of $l$ considered are also specified by the user), the radial order $k$, the period $P_{\text {theo }}\left(=2 \pi / \sigma_{R}\right.$, where $\sigma_{R}$ is the real 
TABLE 3

Pulsation Characteristics of a Model of EC 20117-4014 in the 50-500 s Period WINDOW FOR MODES WITH $l=0,1$, AND 2

\begin{tabular}{|c|c|c|c|c|c|c|}
\hline$l$ & $k$ & $\begin{array}{l}P_{\mathrm{obs}} \\
(\mathrm{s})\end{array}$ & $\begin{array}{l}P_{\text {theo }} \\
(\mathrm{s})\end{array}$ & $\begin{array}{c}\sigma_{I} \\
\left(\operatorname{rad~s}^{-1}\right)\end{array}$ & $\begin{array}{l}\log E \\
\text { (ergs) }\end{array}$ & $C_{k l}$ \\
\hline \multirow[t]{10}{*}{$0 \ldots \ldots \ldots \ldots \ldots$} & 9 & $\ldots$ & 54.272 & $3.75 \times 10^{-4}$ & 39.33385 & $\ldots$ \\
\hline & 8 & $\ldots$ & 58.834 & $2.60 \times 10^{-4}$ & 39.49109 & $\ldots$ \\
\hline & 7 & $\ldots$ & 65.858 & $3.02 \times 10^{-5}$ & 39.83430 & $\ldots$ \\
\hline & 6 & $\ldots$ & 71.865 & $-2.77 \times 10^{-5}$ & 40.22984 & $\ldots$ \\
\hline & 5 & $\ldots$ & 80.646 & $-6.77 \times 10^{-5}$ & 40.25461 & $\ldots$ \\
\hline & 4 & $\ldots$ & 92.799 & $-3.42 \times 10^{-5}$ & 40.63792 & $\ldots$ \\
\hline & 3 & $\ldots$ & 102.028 & $-1.18 \times 10^{-5}$ & 41.05210 & $\ldots$ \\
\hline & 2 & $\ldots$ & 122.222 & $-4.30 \times 10^{-6}$ & 41.25827 & $\ldots$ \\
\hline & 1 & 142.152 & 142.152 & $-8.97 \times 10^{-8}$ & 42.44981 & $\ldots$ \\
\hline & 0 & $\ldots$ & 158.186 & $2.58 \times 10^{-9}$ & 42.54750 & $\ldots$ \\
\hline \multirow[t]{10}{*}{$1 \ldots \ldots \ldots \ldots$} & 10 & $\ldots$ & 53.752 & $4.12 \times 10^{-4}$ & 39.28056 & 0.0037 \\
\hline & 9 & $\ldots$ & 58.501 & $2.85 \times 10^{-4}$ & 39.45732 & 0.0036 \\
\hline & 8 & $\ldots$ & 65.290 & $3.81 \times 10^{-5}$ & 39.84537 & 0.0051 \\
\hline & 7 & $\ldots$ & 70.824 & $-2.52 \times 10^{-5}$ & 40.15206 & 0.0066 \\
\hline & 6 & $\ldots$ & 80.057 & $-6.83 \times 10^{-5}$ & 40.23673 & 0.0063 \\
\hline & 5 & $\ldots$ & 91.262 & $-3.20 \times 10^{-5}$ & 40.65913 & 0.0113 \\
\hline & 4 & $\ldots$ & 100.171 & $-1.72 \times 10^{-5}$ & 40.90283 & 0.0118 \\
\hline & 3 & $\ldots$ & 121.345 & $-4.50 \times 10^{-6}$ & 41.24871 & 0.0127 \\
\hline & 2 & 137.280 & 137.281 & $-1.84 \times 10^{-7}$ & 42.27309 & 0.0271 \\
\hline & 1 & 157.447 & 157.447 & $3.10 \times 10^{-11}$ & 42.49224 & 0.0176 \\
\hline \multirow[t]{12}{*}{$2 \ldots \ldots \ldots \ldots \ldots$} & 10 & $\ldots$ & 53.072 & $4.90 \times 10^{-4}$ & 39.18666 & 0.0034 \\
\hline & 9 & $\cdots$ & 58.056 & $3.13 \times 10^{-4}$ & 39.42177 & 0.0035 \\
\hline & 8 & $\ldots$ & 64.307 & $4.97 \times 10^{-5}$ & 39.86690 & 0.0056 \\
\hline & 7 & $\ldots$ & 69.560 & $-1.99 \times 10^{-5}$ & 40.03640 & 0.0062 \\
\hline & 6 & $\ldots$ & 79.145 & $-6.59 \times 10^{-5}$ & 40.22643 & 0.0071 \\
\hline & 5 & $\ldots$ & 88.562 & $-3.05 \times 10^{-5}$ & 40.66319 & 0.0147 \\
\hline & 4 & $\ldots$ & 98.160 & $-2.52 \times 10^{-5}$ & 40.75163 & 0.0119 \\
\hline & 3 & $\ldots$ & 119.057 & $-4.46 \times 10^{-6}$ & 41.27494 & 0.0217 \\
\hline & 2 & $\ldots$ & 130.056 & $-7.74 \times 10^{-7}$ & 41.81921 & 0.0388 \\
\hline & 1 & $\ldots$ & 156.242 & $-5.34 \times 10^{-9}$ & 42.42437 & 0.0198 \\
\hline & 0 & $\ldots$ & 210.299 & $2.99 \times 10^{-11}$ & 45.95541 & 0.4634 \\
\hline & 1 & $\ldots$ & 436.498 & $3.64 \times 10^{-13}$ & 47.62800 & 0.0653 \\
\hline
\end{tabular}

part of the complex eigenfrequency), the stability coefficient $\sigma_{I}$ (the imaginary part of the eigenfrequency), the logarithm of the kinetic energy of the mode $\log E$, and the dimensionless firstorder rotation coefficient $C_{k l}$. As is standard, our equilibrium models are perfectly spherical, and each mode specified by $(k, l)$ is $(2 l+1)$-fold degenerate in eigenfrequency.

In the context of asteroseismology and mode identification, the most important parameters computed by the pulsation code are evidently the periods (or frequencies) of the modes determined. Since these are dependent on the four input parameters detailed earlier, their comparison to the oscillations observed can be used to infer the physical properties of the target. Further information is provided by the stability coefficient, the sign of which determines whether or not a given mode is expected to be excited. While a positive value of $\sigma_{I}$ signifies that the mode is damped, a negative value implies instability, i.e., that the mode may reach observable amplitudes. As indicated in Table 3, excited modes in EC 14026 stars are predicted to fall into designated unstable period bands, which generally completely overlap with the oscillations detected. We therefore expect all the periods observed for EC 20117-4014 to fall within the theoretical instability range and can use this as a cross-check for the viability of any optimal model identified. In comparison, the remaining pulsation parameters are of secondary importance for practical applications. The kinetic energy gives a measure of the energy required to excite a mode to a given amplitude at the surface and provides some indication as to where in the star it is primarily formed, while the rotation coeffi- cient is useful for interpreting the fine-frequency structure caused by the lifting of the $(2 l+1)$-fold degeneracy in a nonspherical rotating star. The latter is, however, of no immediate interest in the present case, since no rotational splitting was detected in the Fourier transform of the EC 20117-4014 light curves.

\subsection{Finding the Optimal Model(s) for EC 20117-4014}

We thoroughly searched the four-dimensional $\left[T_{\mathrm{eff}}, \log g, M_{*}\right.$, $\log q(\mathrm{H})]$ parameter space for the solution (or solutions) that could optimally reproduce the three oscillations observed for EC 20117-4014 using the Toulouse, as well as the Montréal, numerical package briefly mentioned above. The boundaries of the search domain for the four model parameters were set as follows: $30,000 \mathrm{~K} \leq T_{\text {eff }} \leq 38,000 \mathrm{~K}, \quad 5.60 \leq \log g \leq 6.40, \quad 0.35 \leq$ $M_{*} / M_{\odot} \leq 0.70$, and $-5.20 \leq \log q(\overline{\mathrm{H}}) \leq-2.10$. While the ranges of the effective temperature and surface gravity were loosely based on the spectroscopic estimates of DOD97 (given the uncertainties on the spectroscopic measurements, we allowed for generous intervals), possible values of the other two unknowns rely on stellar evolution theory. According to the evolutionary models of Dorman et al. (1993), the masses of subdwarf B stars are confined to the narrow range $0.40-0.43 \lesssim M_{*} / M_{\odot} \lesssim 0.53$; however, more recent predictions by Han et al. (2002, 2003) suggest a somewhat wider mass distribution. Depending on the formation channel, the total mass of an $\mathrm{sdB}$ can then be as low as $\sim 0.3 M_{\odot}$ or as high as $\sim 0.7 M_{\odot}$, although the distributions are nevertheless peaked around the canonical value of $\sim 0.47 M_{\odot}$. In the case of EC 20117-4014, its wide binary status implies an evolution via the stable Roche lobe overflow channel, which is associated with $\mathrm{sdB}$ masses concentrated toward the higher end of the possible range ( peaking at $\sim 0.51 M_{\odot}$, slightly higher than for the other evolutionary scenarios). We therefore considered it vital to fully explore the higher end of the predicted mass range. Finally, the range of values for $\log q(\mathrm{H})$ was chosen according to the range of envelope masses that characterize evolutionary models of EHB stars.

For the pulsation calculation step, we considered all ( $p$-, $f$-, or $g$-) modes in the 50-500 s range, thus amply spanning the range of the periods observed for EC 20117-4014. With no a priori knowledge on the degree $l$ of any of the three observed modes, some assumption had to be made about their possible values. The usual argument to limit the value of $l$ above which modes are no longer observable is geometric cancellation on the visible disk of the star, which naturally favors values of $l=0,1$, and 2 in pulsating stars in general. However, the mode densities and period distributions seen in several well-observed EC 14026 stars, such as KPD 1930+2752, PG 1605+072, PG 1047+003, PG 0014+ 067 , and PG 1219+534, force one to consider modes with higher $l$-values $(l=3$ and/or 4$)$ as well, at least in those specific instances. Otherwise, there would not be enough theoretical modes available in the period window of interest to simply account qualitatively for the number of observed modes in these cases. Our approach to this problem in the past has been to opt for the simplicity of Occam's razor and pick for the upper limit on $l$ the minimum value that would be sufficient to explain qualitatively the observed mode distribution. For instance, in the case of PG 0014+ 067 (Brassard et al. 2001; Charpinet et al. 2005b), it was explicitly assumed that the observed modes had to belong to degree indices from $l=0$ up to and including $l=3$. The inclusion of $l=3$ was deemed necessary because the observed mode density in PG $0014+067$ is too high to be explained solely in terms of modes with $l=0,1$, and 2 . In contrast, modes with $l=4$ were explicitly ignored because their presence was not needed at the qualitative level. In the case of Feige 48, the period distribution detected 
does not require the inclusion of higher degree modes, and it was decided to model the star only in terms of the canonical values $l=0,1$, and 2 (see Charpinet et al. 2005a). In the present context, the three observed periods can be qualitatively accounted for in terms of modes with $l=0$ and 1 only. However, for good measure, we seek to interpret the observed period distribution of EC 20117-4014 in terms of the same canonical values $l=0,1$, and 2. Of course, we cannot rule out that the observed modes have, in fact, degree values of 3, 4, or even higher, but our approach explicitly excludes this possibility.

As could have been expected from the experience of Charpinet et al. (2005a) with Feige 48, our exploration of parameter space did not reveal well-defined, isolated solutions but, instead, families of solutions. Interestingly, we were not swamped by a plethora of such families, but only a few, and we argue below that one of them is preferable to the others. Each family of solutions is characterized by models that show essentially similar values of the merit function $S^{2}$, and consequently, we cannot discriminate between them on the basis of that function alone. The models belonging to a given family show (very nearly) the same surface gravity, but they may have any value of the effective temperature in the wide range explored, i.e., $30,000 \mathrm{~K} \leq T_{\text {eff }} \leq 38,000 \mathrm{~K}$. The models also show very strong correlations, such that a given value of the total mass and a given value of the $\mathrm{H}$ envelope mass correspond to a given $T_{\text {eff }}$. In that sense, the models of a given family are degenerate. Furthermore, quite clearly, as in the case of Feige 48, an accurate independent spectroscopic measurement of the effective temperature of EC 20117-4014 would be most useful here, precisely to lift such degeneracies. We also note that within a given family of solutions (with only a subtle difference as discussed below), the mode identification remains fixed.

The first family of solutions is characterized by models with $\log g \sim 5.86$. By changing the value of $T_{\text {eff }}$ (within the explored range), the values of $M_{*} / M_{\odot}$ and $\log q(\mathrm{H})$ adjust in such a way in a given family that the goodness-of-fit function $S^{2}$ virtually does not change. In fact, the models in this first family are all able to reproduce the three observed periods within the accuracy of the measurements (see Table 2). This corresponds to values of $S^{2}$ of less than $10^{-6}$ or, equivalently, to an average dispersion of less than $0.0004 \%$ between the observed and computed periods. In comparison, the models of the second family of solutionscharacterized by a surface gravity of $\log g \sim 5.77$-fare less well, with values of $S^{2}$ some 2 orders of magnitude larger than those reached in the first family. While, in an absolute sense, this still represents an excellent fit (a dispersion of only $0.004 \%$ ), the observed periods are not reproduced then within their measured uncertainties. This does not imply that the second solution should be automatically rejected on that basis, because, quite obviously, the constitutive physics that entered our models allows for future upgrades. It could very well be the case that future improved models would favor the second solution. But, of course, we do not know that and we can only argue on the basis of what is currently known. If we take our current models at face value, then this favors the first solution. We can also distinguish a third family of solutions centered on $\log g \sim 5.70$ (there is even a fourth one at still lower gravity), but the quality of fit seriously degrades compared to the first two families, and these extra solutions can be safely discarded on the basis of $S^{2}$ alone in such cases. Quite interestingly, the families of solutions differ in their mode identification in that the radial order $k$ of a given mode simply increments by one unit when going from a high-gravity family to its lowgravity immediate neighbor. This is related to the fact that the periods of modes increase with decreasing surface gravity. We fur- ther note that the three observed periods are never assigned to $l=2$ modes in any of these best-fit families of solutions.

We chose the first family over the second one for two reasons. First, already mentioned just above, is the fact that the observed periodicities are reproduced to within the measurement errors in the first case. Again, this does not imply that the input physics of our models is perfect (as indicated in previous papers of ours, there is, on the contrary, room for improvement at that level), but it does indicate that we can at least reproduce perfectly three observed periods in a given pulsator with the available physics. The second reason, which should be given more weight, is that the estimated surface gravity of EC 20117-4014 is $\log g \sim$ 5.90 , according to DOD97. While the uncertainties are possibly quite large due to the contamination of the spectrum by the mainsequence companion of the system, this estimate is in much better agreement with the value of $\log g \sim 5.86$ characteristic of the first solution than $\sim 5.77$ characteristic of the second one. Conservatively speaking, we cannot completely rule out the second family of solutions on the basis of these arguments. Yet, a choice has to be made, and in the rest of this paper we adopt the highgravity solution as the most reasonable option.

To fully exploit the information contained in the first family of asteroseismological models, it is necessary to specify from the outset the effective temperature, because there are not enough observed periods available to constrain it through the asteroseismological process. For instance, if we assume $T_{\text {eff }}=34,800 \mathrm{~K}$ (a value quite close to the estimated spectroscopic value of $34,898 \mathrm{~K}$ and well within the uncertainties), the optimal model that can perfectly account for the three observed periods must have values of $\log g=5.859, M_{*} / M_{\odot}=0.544$, and $\log q(\mathrm{H})=$ -4.153 . The question of how "superior" is that particular model compared to others in parameter space can be qualitatively answered by examining the behavior of the goodness-of-fit merit function $S^{2}$ in its vicinity. It is not obvious to describe a four-dimensional hypersurface, but we attempted to illustrate the main characteristics of the $S^{2}$ function through Figures 6 and 7.

Figure 6 shows $S^{2}$ contours in the $\log g-T_{\text {eff }}$ domain obtained by optimizing the period match in parameter space when varying the surface gravity and the effective temperature in the intervals indicated and when varying the $\mathrm{H}$ layer mass over the full range of interest, i.e., $-5.20 \leq \log q(\mathrm{H}) \leq-2.10$, but keeping the total mass of the models fixed to the value of the above optimal model, i.e., $M_{*} / M_{\odot}=0.544$. Regions of hyperspace identified with the best fits (lowest values of $S^{2}$ ) appear in dark blue, while areas corresponding to the worst matches to the observed periods are indicated in red. The location of the optimal model is indicated by a red cross. We refer to such a map as a "projected" $S^{2}$ map because, for each grid point in the $\log g-T_{\text {eff }}$ plane, we searched for the smallest value of the merit function by varying the H layer mass. Hence we "projected" the best local value of $S^{2}$ onto the plane. For instance, the number indicated along the white scale in the figure gives the value of $\log q(\mathrm{H})$ that minimizes $S^{2}$ at a number of specific $\log g-T_{\text {eff }}$ points (see below). A similar map is shown in Figure 7, where, this time, $S^{2}$ is found at each $\log g-T_{\text {eff }}$ grid point by varying the total mass of the models over the full range of interest, i.e., $0.35 \leq M_{*} / M_{\odot} \leq 0.70$, but keeping the $\mathrm{H}$ layer mass at a fixed value of $\log q(\mathrm{H})=-4.153$. We emphasize that the main objective of these figures is to examine the behavior of the $S^{2}$ function in the vicinity of the optimal model defined above [i.e., once again, the model with $T_{\text {eff }}=$ $34,800 \mathrm{~K}, \log g=5.859, M_{*} / M_{\odot}=0.544$, and $\log q(\mathrm{H})=$ $-4.153]$, but the plots have other pedagogical values as well. 


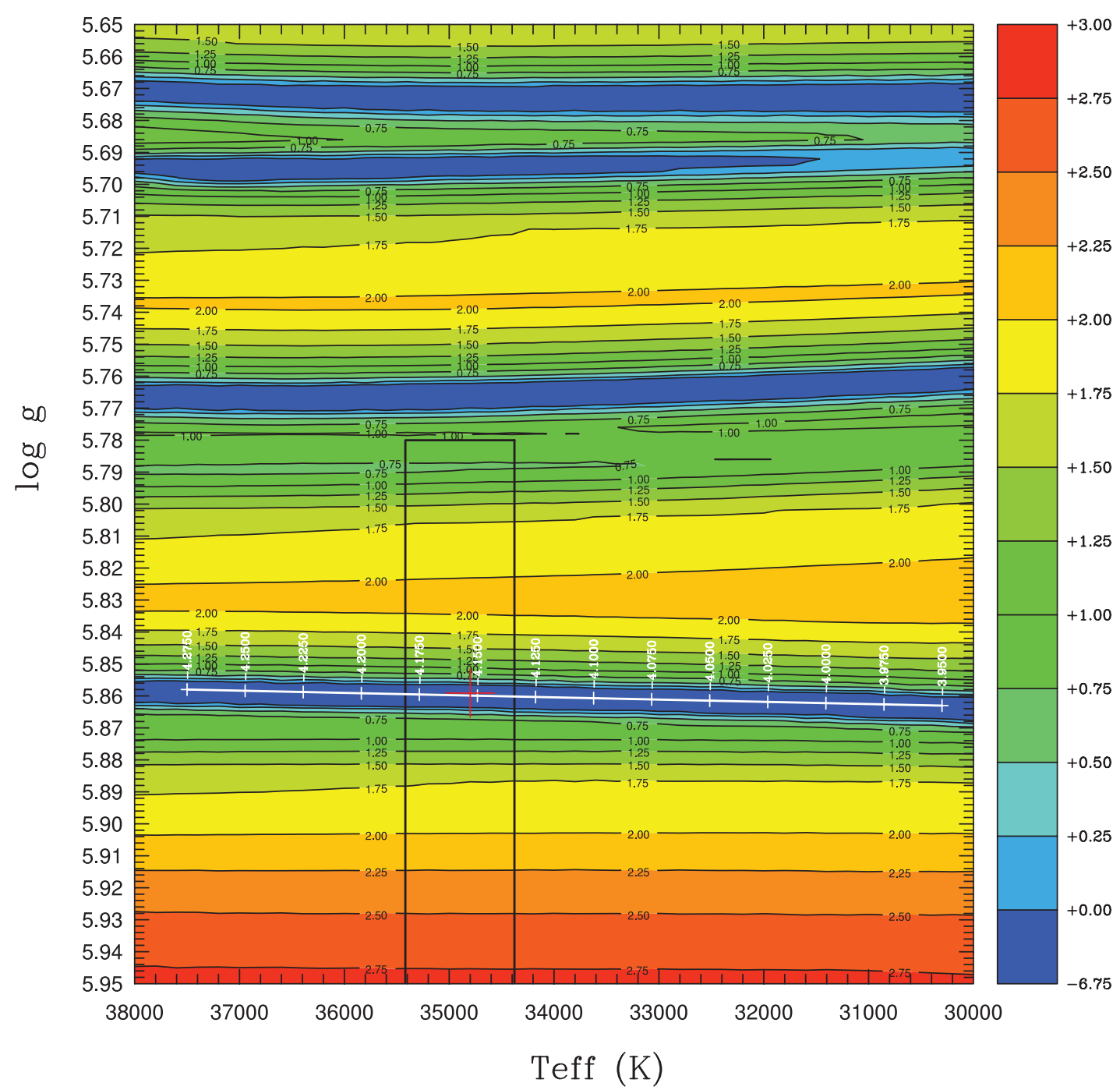

FIG. 6.-Slice of the "projected" $S^{2}$ function (in logarithmic units) along the $\log g-T_{\text {eff }}$ plane with $M_{*}$ set to its optimal value $\left(M_{*}=0.544 M_{\odot}\right)$. In this figure, the projected $\log q(\mathrm{H})$ parameter varies from -5.2 to -2 .1. Its values found at various locations at the bottom of the dark blue valley defining the family of best-fit models are indicated by the labels on the white projection axis. The latter give the location of the $S^{2}$ minimum in the $\log g-T_{\text {eff }}$ plane as a function of the hydrogen shell thickness. The rectangle shows the uncertainty box associated with the spectroscopic estimates of $\log g$ and $T_{\text {eff }}$.

For instance, Figure 6 clearly indicates the presence of the four families of solutions of approximately constant surface gravity already alluded to above and defined in the plot by the dark blue valleys. Bearing in mind the logarithmic scale of the contours, it is obvious that the minima of $S^{2}$ associated with these families of best-fitting models are quite sharp and recreate the measured frequency spectrum far better than an average model. For the reasons given above, however, we believe that the better solution for explaining the three observed periodicities in EC $20117-4014$ is to be found in the high-gravity valley. Note that by fixing the total mass of the models at the value of the optimal model defined above (which belongs to that high-gravity family), Figure 6 does not do full justice to the other families in that their contours are not as well defined and are, in fact, somewhat distorted. This phenomenon is particularly evident in Figure 7, where the choice of $\log q(\mathrm{H})=-4.153$ partially masks the presence of the other families of solutions, since those require significantly different values of that parameter to minimize $S^{2}$. Note also that, in both figures, the domains of $\log g-T_{\text {eff }}$ illustrated are somewhat smaller than the full ranges in which asteroseismological solutions were actually sought. In particular, in this context note how the $S^{2}$ hypersurface forms an increasingly steep wall-like structure when increasing $\log g$ in Figures 6 and 7. This behavior is related to a previous remark of ours, namely, that the density of pulsation modes decreases significantly at high $\log g$ values in sdB star models to the point where we run out of theoretical periods. Hence, we found no point in illustrating that range of surface gravity, from 5.95 to 6.40 , where the period match becomes particularly bad.

Let us now concentrate on the high-gravity family of solutions in Figures 6 and 7. The figures illustrate neatly what was said in words above. In the case of EC 20117-2014, asteroseismology provides a very well defined and accurate value of $\log g$, but it cannot pin down the effective temperature and, along with it, the total mass and the $\mathrm{H}$ envelope mass, because the models belonging to a given family are degenerate in these parameters. In a figurative sense, the dark blue valley has a flat bottom along the temperature direction. Any value of $T_{\mathrm{eff}}$ in the range illustrated leads to a model that explains equally well the three observed periods, provided that the "correct" values of the other parameters are associated with that temperature. These correct values are very nearly those shown in the figures, particularly by the white scales for $\log q(\mathrm{H})$ and $M_{*} / M_{\odot}$. The numbers illustrated are not exact, because it is impossible to represent a four-dimensional 


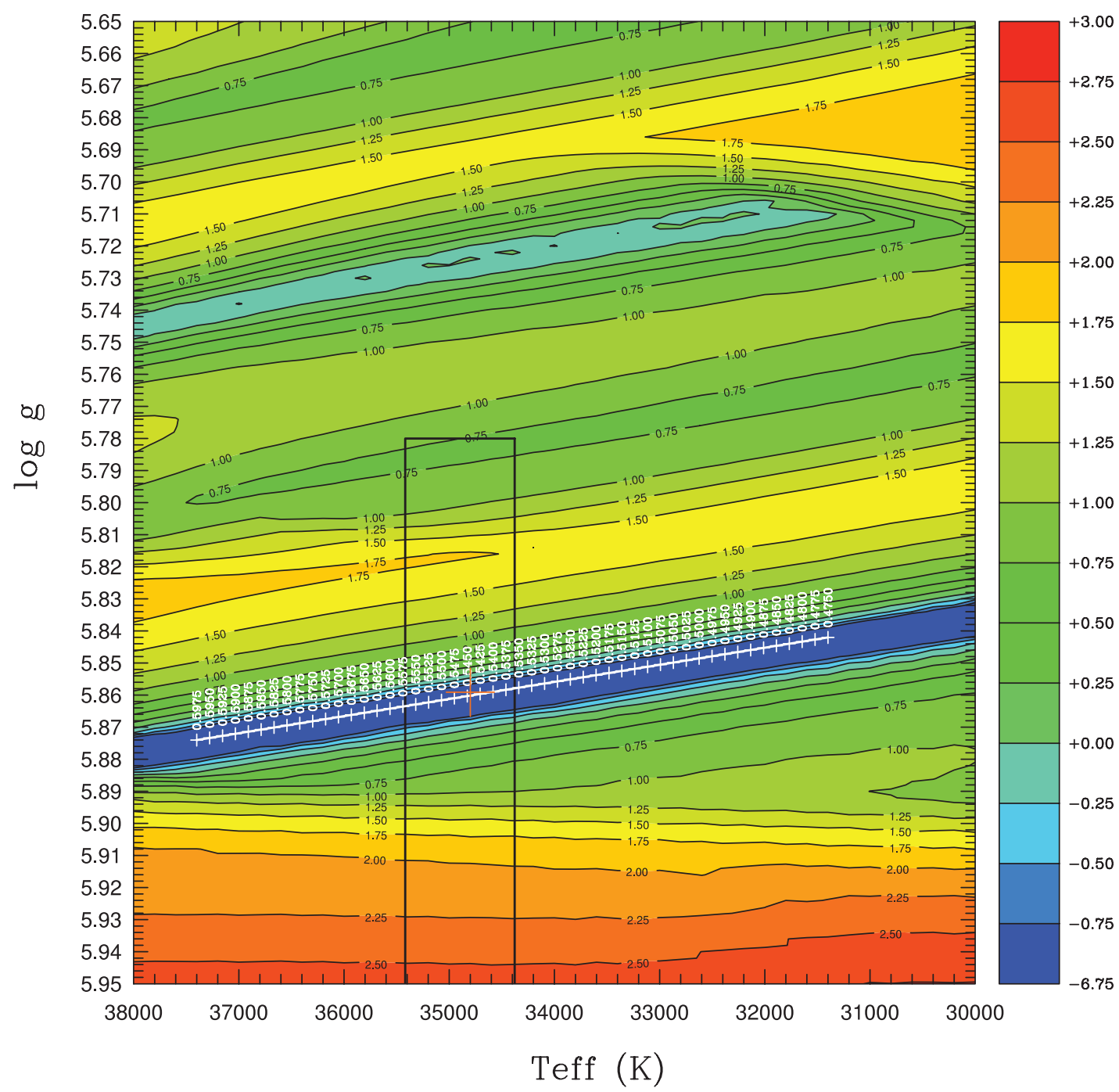

FIG. 7.- Slice of the "projected" $S^{2}$ function (in logarithmic units) along the $\log g-T_{\text {eff }}$ plane with $\log q(\mathrm{H})$ set to its optimal value [ $\left.\log q(\mathrm{H})=-4.153\right]$. In this figure, the projected $M_{*}$ parameter varies from 0.35 to 0.70 . Its values found at various locations at the bottom of the dark blue valley defining the family of best-fit models are indicated by the labels on the white projection axis. The latter give the location of the $S^{2}$ minimum in the $\log g-T_{\text {eff }}$ plane as a function of the total mass. The rectangle shows the uncertainty box associated with the spectroscopic estimates of $\log g$ and $T_{\text {eff }}$.

hypersurface in terms of three-dimensional plots, but they are sufficiently close to the correct values to allow reasonable estimates of the uncertainties of the inferred parameters if one has at one's disposal an accurate and independent estimate of the effective temperature. To demonstrate this, we added a "spectroscopic box" in both Figures 6 and 7, based on the weighted values of $\log g$ (5.90) and $T_{\text {eff }}(34,898 \mathrm{~K})$ obtained previously from the spectroscopic analysis of DOD97 for EC 20117-4014 by using as an illustrative case uncertainties corresponding to twice $(2 \sigma)$ the formal errors of the weighted averages. If we were to adopt these estimates at face value, we would first find that the asteroseismological value of $\log g$ is certainly consistent with the spectroscopic estimate (which is not surprising, since we already used that argument in favor of the high-gravity solution!). More interestingly, we would use the spectroscopic temperature and its associated uncertainties directly to infer the appropriate values of the total mass and the $\mathrm{H}$ envelope mass, as well as their related uncertainties, as a reading of Figures 6 and 7, augmented with the spectroscopic box, clearly implies. In essence, this is what Charpinet et al. (2005a) did in the case of Feige 48.

Unfortunately, in the case of EC 20117-4014, the contamination of the spectrum by a main-sequence companion casts a shadow on the accuracy with which the atmospheric parameters could be inferred from spectroscopy, and we must recognize that the uncertainties used to draw the spectroscopic box in Figures 6 and 7 most likely largely underestimate the true errors. To circumvent this difficulty and avoid possible overinterpretation, we adopt a conservative point of view and assume from now on that the effective temperature of EC 20117-4014 is to be found in a rather wide interval from 32,800 to $36,800 \mathrm{~K}$, i.e., within $\pm 2000 \mathrm{~K}$ of the "central" value of $34,800 \mathrm{~K}$ used previously in the definition of a representative optimal model. All models found in that wide range of $T_{\text {eff }}$ and belonging to the high-gravity family of solutions provide equally valid period matches (same values of $S^{2}$ ) to the three observed periodicities. A detailed examination of our asteroseimological data reveals that if we fix the effective temperature at $T_{\text {eff }}=32,800 \mathrm{~K}$, the associated optimal model must have the values $\log g=5.848, M_{*} / M_{\odot}=0.504$, and $\log q(\mathrm{H})=$ -4.099 . Likewise, at the other extreme, if we fix the effective temperature at $T_{\text {eff }}=36,800 \mathrm{~K}$, the associated optimal model must have the values $\log g=5.864, M_{*} / M_{\odot}=0.585$, and $\log q(\mathrm{H})=-4.250$. These exact values of $M_{*} / M_{\odot}$ and $\log q(\mathrm{H})$ for those two effective temperatures are not precisely equal to those that can be read on the white scales in Figures 6 and 7, but 


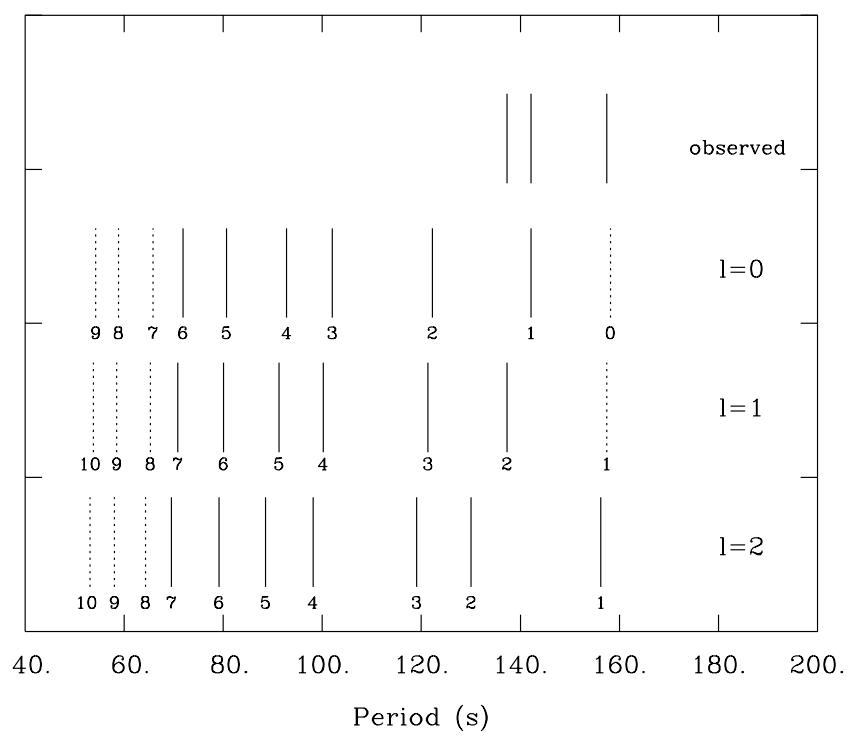

Fig. 8. - Comparison of the observed period spectrum for EC 20117-4014 with the theoretical pulsation spectrum of the $34,800 \mathrm{~K}$ optimal model in the $50-200$ s period range for degree indices $l=0,1$, and 2 . Solid line segments indicate modes predicted to be excited, while dotted line segments refer to stable modes. The radial indices $k$ of the predicted modes are also given.

they are sufficiently close to demonstrate the viability of the graphic method that we used in the figures.

\subsection{Period Fit and Mode Identification}

The pulsational properties of the representative optimal model with $T_{\text {eff }}=34,800 \mathrm{~K}$ are listed in Table 3, together with the observed periods used in the search (also see Fig. 8 for a graphical illustration). We can see that the match achieved is essentially perfect, there being no residuals in the fit at the accuracy of the observational errors. This is not the case, however, for targets involving more than three periods and, as we find out, the average relative dispersion currently achieved from the asteroseismology of EC 14026 stars increases with the number of pulsations detected. This points to inaccuracies in the models that have yet to be resolved. Nevertheless, we believe the fact that our secondgeneration structures have so far been able to recreate the period spectra detected for five short-period variables to an average accuracy of typically $\leqslant 1 \%$ indicates that the basic input physics is fundamentally correct, if not numerically perfect.

Comparable fits are obtained for optimal models with $T_{\text {eff }}=$ 32,800 and $36,800 \mathrm{~K}$ belonging to the same family, but there is a subtle change in mode identification as the effective temperature is varied in that interval. This change is related to the fact that the $l=0, k=0$ mode has a period quite close to that of the mode with $l=1, k=1$ in that family of models and, depending on the value of $T_{\mathrm{eff}}$, the optimization procedure will assign the observed $157.447 \mathrm{~s}$ period to one or the other of these theoretical modes. The situation is summarized in Table 4, where we indicate the mode identification that we obtained for three different values of the effective temperature. For the higher temperatures the optimization method favors the radial mode interpretation for the $157.447 \mathrm{~s}$ pulsation, while for the lower temperatures it favors the dipole mode interpretation. It is not clear at all why only one of these two closely spaced periods should be detectable, but we recall that our photometry was rather limited in terms of the signal-to-noise ratio. Maybe a search at higher sensitivity than was possible in our observations could reveal the existence of a low-amplitude companion period very close to the $157.447 \mathrm{~s}$
TABLE 4

Mode Identification and Stability

\begin{tabular}{clll}
\hline \hline $\begin{array}{c}\text { Period } \\
\text { (s) }\end{array}$ & $T_{\text {eff }}=32,800 \mathrm{~K}$ & $T_{\text {eff }}=34,800 \mathrm{~K}$ & $T_{\text {eff }}=36,000 \mathrm{~K}$ \\
\hline $137.280 \ldots \ldots \ldots .$. & $l=1, k=2,(\mathrm{U})$ & $l=1, k=2,(\mathrm{U})$ & $l=1, k=2,(\mathrm{~S})$ \\
$142.152 \ldots \ldots \ldots . .$. & $l=0, k=1,(\mathrm{U})$ & $l=0, k=1,(\mathrm{U})$ & $l=0, k=1,(\mathrm{~S})$ \\
$157.447 \ldots \ldots \ldots .$. & $l=1, k=1,(\mathrm{U})$ & $l=1, k=1,(\mathrm{~S})$ & $l=0, k=0,(\mathrm{~S})$ \\
\hline
\end{tabular}

mode, or maybe such a mode is simply not excited, for an unknown reason.

Table 4 also reveals the stability condition of each mode derived from our nonadiabatic calculations. A mode labeled with the letter "U" (for "unstable") is excited in our computations and may therefore show a detectable amplitude. Conversely, a mode labeled with the letter "S" (for "stable") is not driven and, consequently, is not expected to be observed. Interestingly, the stability boundary for the modes of interest enters into the range of effective temperature indicated here. Tests indicate that all three modes become unstable only below $T_{\text {eff }}=34,700 \mathrm{~K}$, so this value could be taken as an upper limit on the effective temperature of EC 20117-4014, that is, if we take our nonadiabatic calculations at face value. In this scenario, EC 20117-4014 cannot be as hot as $36,800 \mathrm{~K}$, say, because the appropriate model at that temperature cannot drive pulsation modes. Hence, we could use nonadiabatic arguments to further constrain our possible asteroseismological solutions for EC 20117-4014. In keeping with our conservative approach, however, we refrain from doing that, but we point out here the potential of the method.

In the mode identification achieved, the observed oscillations correspond to low-order $p$-modes with degree indices of $l=0$ and 1 , even though the possibility that these modes could have had $l=2$ as well was explicitly considered. As indicated above, in our best-fit families of possible solutions, the optimization method led naturally to the value of $l=0$ or 1 , even though the a priori choices included $l=0,1$, and 2 . We consider this a robust result. Furthermore, at the outset, it was not clear that the periodicities detected would cluster at the low radial order (or high period) end of the instability band (see Table 3 and Fig. 8 for an illustration of such bands of unstable periods). Incidentally, this is precisely what was found for the other EC 14026 stars analyzed to date. A possible explanation is that, within the band of excited periods, the energy driving the pulsations may preferentially be distributed among modes of low radial order (Charpinet et al. 2005c). The latter would then reach observable amplitudes more easily and therefore be detected more readily. However, lacking nonlinear pulsation theory, it is not clear why certain parts of the unstable period range should be excited to a larger extent than others. In this context it is interesting to note that a similar effect has been observed for some of the long-period variable subdwarf B stars (see, e.g., Randall et al. 2006). In their case nonadiabatic computations are unable to quantitatively account for the ranges of periods observed, but nevertheless theory does predict bands of excited periods, within which all modes should be unstable. These are indeed detected over some of the observed period range; however, we have found significant gaps in the period distribution, which point toward some kind of mode selection taking place. It therefore seems that the channeling of energy into specific frequency bands is intrinsic to both types of pulsating subdwarfs, an idea that should be investigated in more detail in the future.

We should mention that the mode identification postulated here can in principle be tested using an independent means. 
One promising avenue is the exploitation of a mode's coloramplitude dependence on its degree index through multicolor photometry (see, e.g., Randall et al. 2005). Some very encouraging applications of this technique have been published by Jeffery et al. (2004), suggesting the identification of a few modes in two EC 14026 pulsators. Unfortunately, the color-amplitude behavior predicted for modes with $l=0,1$, and 2 is usually very similar, making it very challenging to discriminate between the lowest degree (and thus presumably most visible) modes. Indeed, in a recent study, Tremblay et al. (2006) analyzed quantitatively most of the available multicolor photometry for rapidly pulsating hot B subdwarfs and found that the quality of the data will have to be improved upon markedly if we are to convincingly discriminate between low-degree modes in the EC 14026 stars. However, this is within reach with $4 \mathrm{~m}$ class telescopes. Other ways of restricting the $l$-value for modes observed include the exploitation of rotational splitting (see, e.g., Charpinet et al. 2005a) and line profile variations (see, e.g., Schoenaers \& Lynas-Gray 2006). However, the former is applicable only in the case of sufficiently rapidly rotating pulsators, and the latter has not yet been tested observationally for $\mathrm{sdB}$ pulsators. Thus, for the time being, the analysis of multicolor photometry seems to be the most promising method of verifying the accuracy of the asteroseismological analyses achieved.

\subsection{Structural Parameters of EC 20117-4014}

In our conservative approach, we found that the most practical way of estimating the structural parameters of EC 20117-4014 is simply to use the values of the optimal models found at the extreme ends of the range of effective temperature that we discussed, i.e., $T_{\text {eff }}=32,800$ and $36,800 \mathrm{~K}$. Given this large interval, there is no point in using a more sophisticated type of error analysis, and we adopt the parameters of these two extreme models as credible boundaries in the determination of the actual stellar parameters. The claim is that the "true" parameters should be found somewhere in the intervals of values as summarized in Table 5. We list the primary quantities $\log g, M / M_{\odot}$, and $\log q(\mathrm{H})$ in Table 5, as well as some useful secondary quantities obtained directly from the former (without carrying along various sources of possible errors). Not surprisingly here, the uncertainties on the primary quantities are larger than those inferred by Charpinet et al. (2005a) for Feige 48 on the basis of accurate and reliable optical spectroscopy, giving them an estimate of $T_{\text {eff }}=29,580 \pm 370 \mathrm{~K}$ for that star. For example, our suggested uncertainty on $\log g$ for EC 20117-4014 is almost 3 times that claimed by Charpinet et al. (2005a) for Feige 48. Likewise, the uncertainty on the derived value for the total mass of our target star is some 10 times larger than that obtained for Feige 48.

Despite these limitations, we submit that our results are still highly interesting and, frankly, even surprising, given that we had only three periods to work with. For instance, we find that the value of $\log g$ derived from asteroseismology is consistent with that derived from spectroscopy. Of course, this is partly due to the fact that we selected the $S^{2}$ minimum closest to the spectroscopic gravity to correspond to the family of optimal models. On the other hand, the family chosen did present the best overall fit to the periodicities observed, a result that was by no means guaranteed from the outset. Assuming that the valley picked is the "correct" one and despite the uncertainty in the effective temperature, the surface gravity of EC 20117-4014 is still much more tightly constrained (by a factor of $\sim 3$ ) on the basis of asteroseismology than by the best available spectroscopy. This is due to the strong dependence of the $p$-mode periodicities on the gas density, which is mainly influenced by the $\log g$ parameter. In
TABLE 5

INFERREd Properties of EC 20117-4014

\begin{tabular}{|c|c|c|}
\hline Quantity & $T_{\text {eff }}=32,800 \mathrm{~K}$ & $T_{\text {eff }}=36,800 \mathrm{~K}$ \\
\hline $\log g \ldots$. & 5.848 & 5.864 \\
\hline$M_{*}\left(M_{\odot}\right) \ldots \ldots \ldots \ldots \ldots \ldots$ & 0.504 & 0.585 \\
\hline $\log q(\mathrm{H}) \ldots \ldots \ldots \ldots \ldots \ldots$ & -4.099 & -4.250 \\
\hline$R\left(M_{*}, g\right)\left(R_{\odot}\right) \ldots \ldots \ldots \ldots \ldots$ & 0.140 & 0.148 \\
\hline$L\left(T_{\mathrm{eff}}, R\right)\left(L_{\odot}\right) \ldots \ldots \ldots \ldots \ldots$ & 20.5 & 36.3 \\
\hline$M_{V}\left(g, T_{\mathrm{eff}}, M_{*}\right) \ldots \ldots \ldots \ldots$ & 4.66 & 4.33 \\
\hline$d\left(V, M_{V}\right)(\mathrm{pc}) \ldots \ldots \ldots \ldots \ldots$ & 600 & 698 \\
\hline
\end{tabular}

comparison, the temperature plays a much less important role and is generally determined more accurately from spectroscopy, as pointed out above.

For its part, the total mass inferred for EC 20117-4014, in the range $0.50-0.59 M_{\odot}$, appears to be noticeably higher than those found for the other rapidly pulsating subdwarfs analyzed to date, which tend to cluster around the canonically expected value of $\sim 0.47 M_{\odot}$ (see Charpinet et al. 2006). While this is somewhat surprising, it is not completely incomprehensible from an evolutionary point of view. According to the work of Han et al. (2002, 2003), a subdwarf in a wide binary such as EC 20117-4014 should have formed via a stable Roche lobe overflow. In this scenario, the resulting distribution of $\mathrm{sdB}$ masses is still highly peaked, but there is a high-mass tail, and the peak is located around $0.51 M_{\odot}$, slightly but significantly higher than for other possible evolutionary schemes. Our results presenting evidence for binary motion with a period in the range 20-350 days are indeed highly consistent with the stable Roche lobe overflow scenario. It is clear that the matter remains to be investigated in more detail, particularly considering the uncertainties surrounding the error estimates for the spectroscopy and hence those of the asteroseismological quantities inferred. For instance, if the true effective temperature were to be lower than our conservative lower limit of 32,800 K (and we cannot completely exclude this possibility), then the inferred mass would be more in line with previous determinations. However, for the moment the result obtained for EC 20117-4014 seems to point toward an evolutionary history slightly different from that encountered for the single and short-period binary pulsators analyzed previously. The inferred hydrogen shell thickness, on the other hand, is very similar to that obtained for the hot EC 14026 stars PG 0014+067 and PG $1219+534$ and significantly smaller than that computed for the cooler pulsator Feige 48 (see Charpinet et al. 2005b). This is in line with predictions that subdwarfs with lower effective temperatures should have thicker envelopes, since the latter acts as an isolating layer between the helium core and the stellar surface.

Of all the secondary quantities inferred, only the distance of EC 20117-4014 has previously been estimated. Using the ratio of a stellar radius derived from the spectroscopic value of $\log g$ and an assumed mass of $0.5 M_{\odot}$, as well an apparent angular radius determined from the continuum level of their spectra, DOD97 computed the distance of EC 20117-4014 to be $d=$ $670-680 \mathrm{pc}$. This is in good agreement with the asteroseismological value of $d=600-698$ pc (see Table 5) and as such provides a nice confirmation of the validity of the parameters inferred.

\section{CONCLUSION}

In this study, we obtained further observations of the rapidly pulsating subdwarf B star EC 20117-4014 with the aim of improving on the quality of previous data. Unfortunately, poor weather conditions during both of the main runs prohibited the 
detection of any as yet unknown pulsation periods. We did, however, succeed in measuring a systematic frequency variation over a timescale of a few months, which is most likely due to binary motion. From the constraints posed by the amplitude of the frequency shifts and the lack of rotational splitting in the photometry, we were able to restrict the binary period of the system to between $\sim 20$ and 350 days. According to theoretical evolution calculations, this points to formation of the subdwarf via a stable Roche lobe overflow.

In principle, the evolution of a subdwarf can be constrained from its internal parameters, obtainable through asteroseismological means. We therefore attempted an in-depth analysis of EC 20117-4014 on the basis of the three periods detected. Under the explicit assumption that these modes had to belong to degree indices $l=0,1$, or 2 , we thoroughly searched the fourdimensional parameter space defining our equilibrium stellar structures $\left[T_{\mathrm{eff}}, \log g, M_{*}, \log q(\mathrm{H})\right]$ for models that could precisely account for the three observed periodicities. As in the case of Feige 48 discussed by Charpinet et al. (2005a), the number of observed periods (three in the present case) was too low to pin down a best-fit four-dimensional model, but, perhaps surprisingly, only two families of possible solutions were found by that asteroseismological exercise. Each family of solutions is characterized by a nearly constant gravity and highly correlated relations between the three other parameters, leading to degenerate models. Within a family of solutions, all of these degenerate models explain equally well the three observed periods, so one cannot discriminate between the models on the basis of the goodness-of-fit merit function $S^{2}$ alone. Furthermore, no constraint could be obtained on $T_{\text {eff }}$, as the extent of the families of optimal models was found to cover completely the range specifically investigated, i.e., $30,000 \mathrm{~K} \leq T_{\text {eff }} \leq 38,000 \mathrm{~K}$. However, following Charpinet et al. (2005a), we used external constraints from optical spectroscopy to estimate the effective temperature and deduce, through the strong correlations of the parameters in the family of degenerate models, the total mass and the $\mathrm{H}$ envelope mass. Unfortunately, the contamination of the optical spectrum of EC 20117-4014 prevented us, unlike Charpinet et al. (2005a), who could use a reliable and accurate spectroscopic value for $T_{\text {eff }}$, from carrying out this exercise at the desired level of accuracy. While the work of DOD97 indicated an effective temperature of around $34,800 \mathrm{~K}$, the uncertainties on this remained very hard to quantify. In the end, to remain conservative, we assumed that the true effective temperature of EC 20117-4014 lies somewhere in the rather wide range $32,800-36,800 \mathrm{~K}$.

Despite these limitations, we believe that our study of EC 20117-4014 is useful, and our best estimates of the structural parameters of this star are given in Table 5. Note that these numbers are explicitly based on our choice of the "high-gravity" family of solutions, which for the reasons given in the text above, is to be preferred over the "low-gravity" family. If this is indeed the right choice and if we take the data of Table 5 at face value, then it is interesting to recall that the value of $\log g$ is determined to an accuracy of about a factor of 3 higher than is currently possible from the best available spectroscopy. In addition, asteroseismology allows the estimation of the two structural parameters $M_{*}$ and $\log q(\mathrm{H})$, which normally cannot be inferred any other way. In exceptional cases, the mass can be constrained from radial velocity observations in a binary system; however, the resulting uncertainties are typically very large. The determination of the envelope thickness for its part is a pure product of asteroseismology. We find that our solution leads to a relatively thin hydrogen shell typical of the hotter EC 14026 pulsators so far analyzed, in line with the theory that the cooler subdwarfs should be associated with the thickest envelopes. On the other hand, the mass inferred appears interestingly high. We suggest that this could be a result of EC 20117-4014's evolutionary history, likely to be related to a stable Roche lobe overflow.

The family of optimal models found accounts for the pulsations observed in terms of low-degree modes $(l=0$ and 1 , even though the possibility $l=2$ was also explicitly considered) that cluster at the low radial order end of the theoretical unstable frequency band. Given that a similar effect was encountered for the other sdB pulsators monitored to date, this points to some kind of mode selection mechanism whereby the available pulsation energy is preferentially channeled into the excitation of modes in certain frequency ranges. Unfortunately, current pulsation computations are based on linear theory and are not able to predict the amplitudes of oscillations, leaving us with no means of verifying the theory of selective mode driving.

The fact that we have been able to reproduce the observed periods within the accuracy of their measurements is encouraging, but the basic reason for this success is that we had only a few to fit. In more challenging cases where $\sim 10$ periods must be fitted simultaneously, the average dispersion between the observed and computed periods has turned out to be somewhat less than $1 \%$, much larger than the measurement errors (see Brassard et al. 2001; Charpinet et al. 2003, 2005c). This clearly implies that there is ample room for improvement in our current models of sdB pulsators. At the same time, this success should not be dismissed too summarily, because there was no a priori guarantee that we could reproduce any of the periods observed in EC 20117-4014. It is simply not true, contrary to what we often hear from some members of the community, that with four "free" parameters to build models with, one should be able to reproduce exactly four or fewer observed periods. For instance, if there is a serious error in the computation of the opacity or the equation of state, the computed periods could be totally off, whatever the number of model parameters. As a matter of fact, an example of this situation can be found in the published literature. In their analysis of Feige 48, Reed et al. (2004) had three periods to fit but could only explain two of them; the third one was apparently missing from their theoretical period spectra. These authors conjectured (correctly) that one piece of physics was missing in their models. We proposed that the missing piece of constitutive physics was radiative levitation (see Charpinet et al. [2005a]; Fontaine et al. [2006] for more details).

Given the very limited number of observed periods available, the outstanding result of our study is not so much that we managed to find such a close theoretical match to the measured periods, but rather that we were able to isolate a small number of $S^{2}$ minima in the parameter range suggested by spectroscopy. The fact that it was feasible to isolate just one plausible family of optimal models on the basis of only three observed periods opens up the exciting possibility of deriving the asteroseismological parameters for many more known EC 14026 stars without obtaining extra data at higher sensitivity. It had previously been assumed that the 2-3 oscillations detected in the majority of known EC 14026 stars were completely useless in terms of quantitative asteroseismology. However, it should be kept in mind that the principal reason this exercise was so enlightening for EC 201174014 is its relatively high surface gravity, which implies the theoretical excitation of a relatively sparse period spectrum compared to an average, less compact sdB model. Hence, while asteroseismology on the basis of very few observed frequencies may work well for the odd high-gravity fast pulsator, the majority of these objects will require follow-up monitoring on midsize telescopes. Nonetheless, our results underscore the vast potential of the 
forward approach in asteroseismology, as well as the validity of the iron bump opacity mechanism as the instability driver in EC 14026 stars (Charpinet et al. 1997). Ultimately, we hope asteroseismological quantities will be derived for the greater part of all pulsating subdwarf B stars, thus leading to a mature understanding of their evolutionary status.

S. K. R. would like to thank Allan Willis for generously arranging funding for the SAAO 2001 observing run. Observations at SAAO in 2002 July were made possible through the generosity of the Portuguese Foundation. This work was also supported in part by the Natural Sciences and Engineering Research Council of Canada. G. F. also acknowledges the contribution of the Canada Research Chair Program. S. C. made extensive use of the computing facilities offered by the Calcul en Midi-Pyrénées (CALMIP) project. The authors are indebted to the South African Astronomical Observatory (SAAO) for generous allocations of telescope time and to D. Kilkenny for instruction on the use of the telescopes and instruments.

\section{REFERENCES}

Bergeron, P., Wesemael, F., Beauchamp, A., Wood, M. A., Lamontagne, R., Fontaine, G., \& Liebert, J. 1994, ApJ, 432, 305

Billères, M., Fontaine, G., Brassard, P., Charpinet, S., Liebert, J., \& Saffer, R. A. 2000, ApJ, 530, 441

Billères, M., Fontaine, G., Brassard, P., \& Liebert, J. 2002, ApJ, 578, 515

Brassard, P., \& Fontaine, G. 1994, in IAU Colloq. 147, The Equation of State in Astrophysics, ed. G. Chabrier \& E. Schatzman (Cambridge: Cambridge Univ. Press), 560

Brassard, P., Fontaine, G., Billères, M., Charpinet, S., Liebert, J., \& Saffer, R. A. 2001, ApJ, 563, 1013

Brassard, P., Pelletier, C., Fontaine, G., \& Wesemael, F. 1992, ApJS, 80, 725 Charpinet, S., Fontaine, G., \& Brassard, P. 2001, PASP, 113, 775

- 2003, in White Dwarfs, Proc. 13th European Workshop on White Dwarfs, ed. D. de Martino, R. Silvotti, J.-E. Solheim, \& R. Kalytis (NATO Sci. Ser. II, 105; Dordrecht: Kluwer), 69

Charpinet, S., Fontaine, G., Brassard, P., Billères, M., Green, E. M., \& Chayer, P. 2005a, A\&A, 443, 251

2005b, in ASP Conf. Ser. 334, Proc. 14th European Workshop on White Dwarfs, ed. D. Koester \& S. Moehler (San Francisco: ASP), 619

Charpinet, S., Fontaine, G., Brassard, P., Chayer, P., \& Green, E. M. 2006, Baltic Astron., 15, 305

Charpinet, S., Fontaine, G., Brassard, P., Chayer, P., Rogers, F. J., Iglesias, C. A., \& Dorman, B. 1997, ApJ, 483, L123

Charpinet, S., Fontaine, G., Brassard, P., \& Dorman, B. 1996, ApJ, 471, L103 . 2000, ApJS, 131, 223

Charpinet, S., Fontaine, G., Brassard, P., Green, E. M., \& Chayer, P. 2005c, A\&A, 437, 575

Chayer, P., et al. 2004, Ap\&SS, 291, 359

D’Cruz, N. L., Dorman, B., Rood, R. T., \& O’Connell, R. W. 1996, ApJ, 466, 359

Dorman, B., Rood, R. T., \& O’Connell, R. W. 1993, ApJ, 419, 596

Fontaine, G., Brassard, P., Charpinet, S., Green, E. M., Chayer, P., Billères, M., \& Randall, S. K. 2003, ApJ, 597, 518

Fontaine, G., Brassard, P., Wesemael, F., \& Tassoul, M. 1994, ApJ, 428, L61

Fontaine, G., Charpinet, S., Brassard, P., Chayer, P., Rogers, F. J., Iglesias, C. A., $\&$ Dorman, B. 1998, in IAU Symp. 185, New Eyes To See inside the Sun and Stars, ed. F.-L. Deubner, J. Christensen-Dalsgaard, \& D. W. Kurtz (Dordrecht: Kluwer), 367

Fontaine, G., \& Chayer, P. 1997, The Third Conference on Faint Blue Stars, ed. A. G. D. Philip, J. Liebert, R. Saffer, \& D. S. Hayes (Schenectady: L. Davis), 169
Fontaine, G., Green, E. M., Chayer, P., Brassard, P., Charpinet, S., \& Randall, S. K. 2006, Baltic Astron., 15, 211

Green, E. M., et al. 2003, ApJ, 583, L31

Han, Z., Podsiadlowski, P., Maxted, P. F. L., \& Marsh, T. R. 2003, MNRAS, 341,669

Han, Z., Podsiadlowski, P., Maxted, P. F. L., Marsh, T. R., \& Ivanova, N. 2002, MNRAS, 336, 449

Heber, U. 1986, A\&A, 155, 33

Horne, J. H., \& Baliunas, S. L. 1986, ApJ, 302, 757

Jeffery, C. S., Dhillon, V., Marsh, T., \& Ramachandran, B. 2004, MNRAS, 352, 699

Kilkenny, D. 2002, in IAU Colloq. 185, Radial and Nonradial Pulsations as Probes of Stellar Physics, ed. C. Aerts, T. R. Bedding, \& J. ChristensenDalsgaard (ASP Conf. Ser. 259; San Francisco: ASP), 356

Kilkenny, D., Koen, C., O'Donoghue, D., \& Stobie, R. S. 1997a, MNRAS, 285,640

Kilkenny, D., O’Donoghue, D., Koen, C., Stobie, R. S., \& Chen, A. 1997b, MNRAS, 287, 867

Koen, C., Kilkenny, D., O’Donoghue, D., Van Wyk, F., \& Stobie, R. S. 1997, MNRAS, 285, 645

Lasker, B. M., Sturch, C. R., McLean, B. J., Russel, J. L., Jenkner, H., \& Shara, M. M. 1990, AJ, 99, 2019

Montgomery, M. H., \& O’Donoghue, D. 1999, Delta Scuti Star Newletter, No. 13

O’Donoghue, D., Lynas-Gray, A. E., Kilkenny, D., Stobie, R. S., \& Koen, C. 1997, MNRAS, 285, 657 (DOD97)

Randall, S. K., Fontaine, G., Brassard, P., \& Bergeron, P. 2005, ApJS, 161, 456

Randall, S. K., et al. 2006, ApJ, 643, 1198

Reed, M. D., et al. 2004, MNRAS, 348, 1164

Schechter, P. L., Mateo, M., \& Saha, A. 1993, PASP, 105, 1342

Schoenaers, C., \& Lynas-Gray, A. E. 2006, Baltic Astron., 15, 219

Silvotti, R., Ostensen, R., Heber, U., Solheim, J.-E., Dreizler, S., \& Altmann, M. 2002, A\&A, 383, 239

Stobie, R. S., Chen, A., O’Donoghue, D., \& Kilkenny, D. 1992, in ASP Conf. Ser. 30, Variable Stars and Galaxies, ed. B. Warner (San Francisco: ASP), 87

Stobie, R. S., Kawaler, S. D., Kilkenny, D., O’Donoghue, D., \& Koen, C. 1997, MNRAS, 285, 651

Sweigart, A. V. 1997, ApJ, 474, L23

Tremblay, P.-E., Fontaine, G., Brassard, P., Bergeron, P., \& Randall, S. K. 2006, ApJ, in press

Unno, W., Osaki, Y., Ando, H., Saio, H., \& Shibahashi, H. 1989, Nonradial Oscillations of Stars (2nd. ed.; Tokyo: Univ. Tokyo Press) 